\title{
A moment-based analytic approximation of the risk-neutral density of American options
}

\section{J. C. Arismendi \& Marcel Prokopczuk}

To cite this article: J. C. Arismendi \& Marcel Prokopczuk (2016) A moment-based analytic approximation of the risk-neutral density of American options, Applied Mathematical Finance, 23:6, 409-444, DOI: 10.1080/1350486X.2017.1297726

To link to this article: https://doi.org/10.1080/1350486X.2017.1297726

\section{Published online: 08 Mar 2017.}

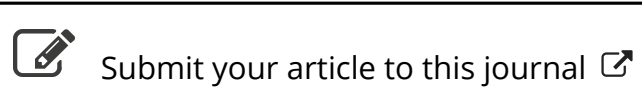

ЦII Article views: 99

View Crossmark data ¿ 


\section{A moment-based analytic approximation of the risk-neutral density of American options}

\section{J. C. Arismendi (1) and Marcel Prokopczuk ${ }^{\mathrm{b}}$}

aICMA Centre - Henley Business School, University of Reading, Whiteknights, Reading, UK; 'b 5 chool of Economics and Management, Leibniz University Hannover, Hannover, Germany

\begin{abstract}
The price of a European option can be computed as the expected value of the payoff function under the risk-neutral measure. For American options and path-dependent options in general, this principle cannot be applied. In this paper, we derive a modelfree analytical formula for the implied risk-neutral density based on the implied moments of the implicit European contract under which the expected value will be the price of the equivalent payoff with the American exercise condition. The risk-neutral density is semi-parametric as it is the result of applying the multivariate generalized Edgeworth expansion, where the moments of the American density are obtained by a reverse engineering application of the least-squares method. The theory of multivariate truncated moments is employed for approximating the option price, with important consequences for the hedging of variance, skewness and kurtosis swaps.
\end{abstract}

\section{ARTICLE HISTORY}

Received 12 September 2014 Accepted 9 February 2017

\section{KEYWORDS}

Multi-asset risk-neutral density; American multi-asset options; higher order moments

One of the most attractive features of Black and Scholes' (1973) work is that they present the value of a contingent claim over a stochastic process with a closed-form formula. In the case of American options, Barone-Adesi and Whaley (1987) provide the first standard analytical approximation. Their approach is based on an iterative algorithm that approximates the early exercise premium. Recent refinements include Kim (1990), Ju (1998) and Ju and Zhong (1999). ${ }^{1}$ In Flamouris and Giamouridis (2002), the implied probability density function of American options is calculated using the procedure of Corrado and $\mathrm{Su}$ (1996), where a univariate Edgeworth expansion is used to fit the probability density function of the option by employing higher order moments. The skewness and kurtosis values are found with a calibration algorithm. In Tian (2011), the univariate implied risk-neutral moments of American options are extracted from option prices with an implied Binomial tree, which is a model-free implied volatility approach originally developed by Breeden and Litzenberger (1978), Rubinstein (1994) and Jackwerth and Rubinstein (1996). ${ }^{2}$

All the above consider univariate cases only. For multi-asset options, several analytical approximations have been developed over recent decades. The first successful model is Margrabe (1978) who derives a closed-form expression for an exchange

CONTACT J. C. Arismendi $\otimes$ j.arismendi@icmacentre.ac.uk $\Theta I C M A$ Centre - Henley Business School, University of Reading, Whiteknights, Reading RG6 6BA, UK 
option. $^{3}$ Carr (1988) extends Margrabe's formula for the case of sequential exchange options. ${ }^{4}$ More recent analytical approximations for the bivariate case (spread options) include Kirk (1996) and Li, Deng and Zhou (2008). Borovkova, Permana and Weide (2007) develop a formula that incorporates the univariate moments. ${ }^{5}$ However, all these attempts to approximate the density of a basket of assets employ the univariate sum of lognormal variables, which results in a substantial loss of information about properties of the multivariate distribution. Moreover, most of the closed-form formulae derived for the European options class are related to the truncation of a moment of the riskneutral density. In the search for a closed-form formulae, a unified theory about how to calculate the option prices using truncated moments has never been used. In this paper, we contribute to the literature in at least three ways. First, we provide an analytical and model-free approximation of the risk-neutral density implied by American multi-asset options, based on the moments of the implied European contract. The analytical approximation is generated with an extension of the multivariate generalized Edgeworth expansion (MGEE) of Arismendi (2014). It is model free, as we do not make any assumption about the generating process of the underlyings. To the best of our knowledge, this is the first time that an analytical approximation for the implied risk-neutral density (IRND) of American multi-asset options has been provided. Similar procedures for extracting the univariate risk-neutral density in the case of European options include Jondeau and Rockinger (2001), Lim, Martin and Martin (2005), Rompolis and Tzavalis (2008) and Rompolis (2010).

Second, as a result of our implementation, non-lognormal risk-neutral densities can be employed. Generally, one can think of two ways for finding an expansion of the multivariate risk-neutral density based on higher order moments: (1) the multi-asset extension of Jarrow and Rudd (1982) and (2) the polynomial expansion of Schlögl (2013). The former makes no assumptions about the form of the risk-neutral density, the latter assumes the risk-neutral density to be given by a Gram-Charlier Type A series expansion, that is, a polynomial expansion around the multivariate normal (MVN) distribution. We follow the former approach for the analytic approximation in our paper as it is more flexible in the auxiliary density selected, providing the possibility for using heavy-tailed and/or skewed distributions. ${ }^{6}$

Using the MGEE, American option implied moments up to the second are obtained by applying a reverse engineering process of the Longstaff and Schwartz (2001) algorithm. The use of only the first two moments is motivated by the empirical work of Jondeau and Rockinger (2000), Zhang and Xiang (2008), and Zhao, Zhang and Chang (2013), who show that the premium associated with higher order moments, that is, skewness and kurtosis, of the risk-neutral density are already determined by the volatility smirk. Finally, the multi-asset option price is computed by integrating the payoff over the multivariate risk-neutral density, relying on an analytical method that extends the multi-asset option approximations of Li, Deng and Zhou (2010) and Alexander and Venkatramanan (2012).

Third, we develop new methods and applications in the area of multivariate truncated moments to find closed-form formulae for options where the underlying processes are multivariate. Our new results allow us to link the mathematical and statistical theory behind truncated moments with the application of option pricing. The new results also allow us to derive multivariate generalizations of univariate option pricing formulae. 
The MGEE is composed of multivariate log-contracts that we define as performance contracts. Log-contracts are associated with the hedging of moment swaps, as demonstrated by Demeterfi et al. (1999) and Schoutens (2005). The resulting MGEE of an American option provides an intuitive formula, where the price of the option is equal to the price of a European option plus the difference in higher order moments given by the early exercise premium. This difference is due to multivariate log-contracts, and closed-form formulae are derived for their pricing employing the multivariate truncated moments framework.

The structure of this paper is as follows: Section 1 presents the notation and the distribution used by the MGEE to approximate the European part of an American option. Section 2 develops the analytical second-order moment approximation. Section 3 derives the algorithm to extract the difference in the moments associated with the early exercise premium. In Section 4, the theory of multivariate truncated moments is developed for pricing multivariate log-contracts associated with the general moments' expansion. In Section 5, a calibration algorithm and numerical results are presented. Finally, Section 6 provides concluding remarks, and further extensions are suggested.

\section{Multi-asset risk-neutral density}

Let $Q$ be the $n$-variate risk-neutral probability measure. Define the filtered probability space $(\Omega, \mathcal{F}, Q)$, where $\mathcal{F}$ is the filtration generated by the sigma-algebra $\left\{\mathbf{S}: \mathcal{F}_{t}=\left\{S_{i}(t), t \geq 0\right\}\right\}$, with the $n$-variate stochastic asset process $\mathbf{S}(t)=$ $\left\{S_{i}(t) \in \mathbb{R}^{+}, t \geq 0\right\}, \quad i \in\{1, \ldots, n\}$. Denote by $g_{\mathbf{S}(t)}$, the density of $\mathbf{S}(t)$ under $Q$. Define $\mathbf{a}=\left(a_{1}, \ldots, a_{n}\right), a_{i} \in \mathbb{R}$ and denote the abbreviated integral operator as

$$
\int_{a_{1}}^{\infty} \ldots \int_{a_{n}}^{\infty}(\cdot) d s_{1}(t) \ldots d s_{n}(t)=\int_{a_{i}}^{(n)}(\cdot) d s(t),
$$

for $i \in\{1, \ldots, n\}$. Let $\Pi(\mathbf{S}(t))$ represent an option payoff function. If $g_{\mathbf{S}(t)}$ is a continuous density function, the price of a European option under the risk-neutral measure $Q$, at $t=0$, can be computed as

$$
\begin{aligned}
C_{E, 0}(\Pi(\mathbf{S}(t))) & =\exp (-r t) \mathbb{E}_{0}^{Q}[\Pi(\mathbf{S}(t))] \\
& =\exp (-r t) \int_{-\infty}^{(n)} \Pi(\mathbf{s}(t)) g_{\mathbf{S}(t)} \mathrm{d} s(t)
\end{aligned}
$$

where $r$ is the risk-free rate assumed to be constant. The notation $\mathbf{s}(t)$ will be used when referring to the variable of integration. Define an American option with the same payoff over the multivariate asset $\mathbf{S}(t)$. The price of the American option is

$$
C_{A, 0}(\Pi(\mathbf{S}(t)))=C_{E, 0}(\Pi(\mathbf{S}(t)))+A_{\varepsilon},
$$

where $A_{\varepsilon}$ is the value of the early exercise premium.

Suppose there exists an $n$-variate process $\mathbf{X}(t)=\left\{X_{i}(t) \in \mathbb{R}^{+}, t \geq 0\right\}$, with continuous density $f_{\mathbf{X}(t)}$, such that the expected value of the payoff function $\Pi(\mathbf{X}(t))$ over this 
process under the risk-neutral measure $Q$ will be equal to the value of the American option:

$$
\begin{aligned}
C_{A, 0}(\Pi(\mathbf{S}(t))) & =C_{E, 0}(\Pi(\mathbf{X}(t))) \\
& =\exp (-r t) \mathbb{E}_{0}^{Q}[\Pi(\mathbf{X}(t))] \\
& =\exp (-r t) \int_{-\infty}^{(n)} \Pi(\mathbf{x}(t)) f_{\mathbf{X}(t)} \mathrm{d} \mathbf{x}(t),
\end{aligned}
$$

where $\Pi(\mathbf{X}(t))$ is equal to $\Pi(\mathbf{S}(t))$, exchanging $\mathbf{S}(t)$ by $\mathbf{X}(t)$.

We are interested in finding a density $f_{\mathbf{X}(t)}$ such that

$$
C_{E, 0}(\Pi(\mathbf{X}(t)))=C_{E, 0}(\Pi(\mathbf{S}(t)))+A_{\varepsilon},
$$

or equivalently,

$$
\int_{-\infty}^{(n)} \Pi(\mathbf{x}(t)) f_{\mathbf{X}(t)} \mathrm{d} \mathbf{x}(t)=\int_{-\infty}^{(n)} \Pi(\mathbf{s}(t)) g_{\mathbf{S}(t)} d \mathbf{s}(t)+A_{\varepsilon} .
$$

The risk-neutral density, $f_{\mathbf{X}(t)}$, will be defined as the IRND of the American option. Equivalently, the value of a European option with payoff $\Pi(\mathbf{X}(t))$ and risk-neutral density $f_{\mathbf{X}(t)}$ will be equal to the American option value $C_{A, 0}(\Pi(\mathbf{S}(t)))$.

There could be different densities $f_{\mathbf{X}(t)}$ that satisfy (1) or (2), but we are interested in finding a density in such a way that it is model free, that is, depends on knowing neither the generating process of $\mathbf{S}(t)$, nor the generating process of $\mathbf{X}(t)$, but only the riskneutral density $g_{\mathbf{S}(t)}$. In this case, there exists an approximation that uses information of $g_{\mathbf{S}(t)}$, and the difference in the cumulants between the risk-neutral density $f_{\mathbf{X}(t)}$ and $g_{\mathbf{S}(t)}$ : the multivariate extension of the Jarrow and Rudd (1982) Edgeworth expansion, termed the MGEE, defined in Arismendi (2014), where $f_{\mathbf{X}(t)}$ is approximated as

$$
f_{\mathbf{X}} \approx g_{\mathbf{S}}+\sum_{j=1}^{2} M_{\left[l_{1},\left[l_{2}\right]\right]} \frac{(-1)^{j}}{j !} \frac{\partial^{j}}{\partial s_{\left[l_{1}\right]} \partial s_{\left[l_{2}\right]}} g_{\mathbf{S}}
$$

where the tensor notation $M_{\left[l_{1},\left[l_{2}\right]\right]} \frac{(-1)^{j}}{j !} \frac{\partial^{j}}{\partial s_{\left[l_{1}\right]}^{\partial s}\left[l_{2}\right]} g_{\mathbf{S}}$ is

$$
M_{\left[l_{1},\left[l_{2}\right]\right]} \frac{(-1)^{j}}{j !} \frac{\partial^{j}}{\partial s_{\left[l_{1}\right]} \partial s_{\left[l_{2}\right]}} g_{\mathbf{s}}=\sum_{l_{1}=1}^{n}\left(M_{l_{1}}(-1) \frac{\partial}{\partial s_{l_{1}}} g_{\mathbf{S}}+\sum_{l_{2}=1}^{n}\left(M_{l_{1}, l_{2}}\left(\frac{1}{2}\right) \frac{\partial^{2}}{\partial s_{l_{1}} \partial s_{l_{2}}} g_{\mathbf{s}}\right)\right),
$$

and $M_{l_{1}}, M_{l_{1}, l_{2}}$ are the differences of the first- and second-order moments of the riskneutral densities $f_{\mathbf{X}(t)}$ and $g_{\mathbf{S}(t)}$. The left-hand side in (3) is equal to the right-hand side when the error of the approximation $\varepsilon(\mathbf{s}, 3)$ is zero:

$$
\varepsilon(\mathbf{s}, 3)=\sum_{j=3}^{\infty} M_{\left[l_{1},\left[l_{2},\left[\ldots,\left[l_{j}\right] \ldots\right]\right.\right.} \frac{(-1)^{j}}{j !} \frac{\partial^{j}}{\partial s_{\left[l_{1}\right]} \ldots \partial s_{\left[l_{j}\right]}} g_{\mathbf{s}}=0
$$

Zhao, Zhang and Chang (2013) point out that the premium associated with higher order moments above the second is contained in the volatility smirk. Thus, we could calibrate $M_{\left[l_{1},\left[l_{2}\right]\right]}$ such that the equality in (3) holds. As a consequence, (4) holds and a 
result of (4) would be that the early exercise premium is equal to the option price premium of the cumulants' differences of $f_{\mathbf{X}(t)}$ and $g_{\mathbf{S}(t)}$ :

$$
A_{\varepsilon}=\sum_{j=1}^{2} C_{E, 0, \mathbb{W},\left[l_{1}, \ldots, l_{j}\right]}(\Pi(\mathbf{S}(t))),
$$

where

$$
\begin{aligned}
\sum_{j=1}^{2} C_{E, 0, \mathbb{W},\left[l_{1}, \ldots, l_{j}\right]}(\Pi(\mathbf{s}(t)))= & \exp (-r t) \sum_{l_{1}=1}^{n} \\
& \left(\begin{array}{l}
M_{l_{1}}(-1) \int_{-\infty}^{(n)} \Pi(\mathbf{s}(t)) \frac{\partial}{\partial s_{l_{1}}} g_{\mathbf{s}} d \mathbf{s}(t) \\
+\sum_{l_{2}=1}^{n}\left(M_{l_{1}, l_{2}}\left(\frac{1}{2}\right) \int_{0}^{(n)} \Pi(\mathbf{s}(t)) \frac{\partial^{2}}{\partial s_{l_{1}} \partial s_{l_{2}}} g_{\mathbf{s}} d \mathbf{s}(t)\right)
\end{array}\right) .
\end{aligned}
$$

There are two ways of using (3):

(1) The density $g_{\mathbf{S}(t)}$ is known, the cumulants' differences $M_{\left[l_{1},\left[l_{2}\right]\right]}$ are estimated, then we can generate an implied density $f_{\mathbf{X}(t)}$.

(2) The density $f_{\mathbf{X}(t)}$ and the cumulants' differences $M_{\left[l_{1},\left[l_{2}\right]\right]}$ are known, then we could approximate $g_{\mathbf{S}(t)}$.

Our problem is the first one. The difference of the moments can be expressed in terms of the difference of cumulants of $f_{\mathbf{X}(t)}$ and $g_{\mathbf{S}(t)}$ as

$$
\begin{gathered}
M_{l_{1}}=k_{l_{1}}\left(f_{\mathbf{X}(t)}\right)-k_{l_{1}}\left(g_{\mathbf{S}(t)}\right), \\
M_{l_{1}, l_{2}}=\left(k_{l_{1}, l_{2}}\left(f_{\mathbf{X}(t)}\right)-\lambda_{\operatorname{VCF}(2)} k_{l_{1}, l_{2}}\left(g_{\mathbf{S}(t)}\right)\right)+M_{l_{1}} M_{l_{2}},
\end{gathered}
$$

where $\lambda_{\mathrm{VCF}(2)}$ is a calibration parameter to obtain the equality in (5), incorporating the error difference (4) into the IRND. The cumulants $k_{l_{1}}\left(g_{\mathbf{S}(t)}\right)$ and $k_{l_{1}, l_{2}}\left(g_{\mathbf{S}(t)}\right)$ can be calculated from the density $g_{\mathbf{S}(t)}$ that is provided. The cumulants $k_{l_{1}}\left(f_{\mathbf{X}(t)}\right)$ and $k_{l_{1}, l_{2}}\left(f_{\mathbf{X}(t)}\right)$ will be estimated in Section 3.

\section{The analytic approximation}

Let the process $\mathbf{S}(t)$ be defined as

$$
\begin{aligned}
d S_{i}(t)= & \mu_{i} S_{i}(t) \mathrm{d} t+\sigma_{i} S_{i}(t) \mathrm{d} W_{i}(t), \\
& \left\langle\mathrm{d} W_{i}(t), \mathrm{d} W_{j}(t)\right\rangle=\rho_{i, j} \mathrm{~d} t,
\end{aligned}
$$

where $i, j \in\{1, \ldots, n\}, \mu_{i}, \sigma_{i}$ are the constant ${ }^{7}$ risk-neutral mean and the constant volatility of the variable $S_{i}(t), W_{i}(t)$ are Wiener processes under the risk-neutral measure $Q$ and $\rho_{i, j}$ is the constant correlation between $S_{i}(t)$ and $S_{j}(t)$. 
Let the initial values be $\mathbf{S}(0)=\left(S_{1}(0), \ldots, S_{n}(0)\right)$. Define the vector $\log (\mathbf{S}(t))=\left(\log \left(S_{1}(t)\right), \ldots, \log \left(S_{n}(t)\right)\right)$. Applying Itô's Lemma to each component of $\log (\mathbf{S}(t))$, and solving this differential equation, we have

$$
\log \left(S_{i}(t)\right)=\log \left(S_{i}(0)\right)+\left(r-\frac{1}{2} \sigma_{i}^{2}\right) t+\sigma_{i} W_{i}(t) .
$$

The distribution $g_{\mathbf{S}}(t)$ will be $n$-variate lognormal with parameters:

$$
\mu_{\mathbf{s}}=\left(\begin{array}{c}
\log \left(S_{1}(0)\right)+\left(r-\frac{1}{2} \sigma_{1}^{2}\right) t \\
\vdots \\
\log \left(S_{n}(0)\right)+\left(r-\frac{1}{2} \sigma_{n}^{2}\right) t
\end{array}\right), \quad \Sigma_{\mathbf{s}}=\left(\begin{array}{ccc}
\sigma_{1}^{2} t & \sigma_{1} \sigma_{2} \rho_{1,2} t & \cdots \\
\sigma_{2} \sigma_{1} \rho_{1,2} t & \sigma_{2}^{2} t & \cdots \\
\vdots & & \ddots
\end{array}\right)
$$

where the vector $\log (\mathbf{S}(t))=\left(\log \left(S_{1}(t)\right), \ldots, \log \left(S_{n}(t)\right)\right)$.

Define by $\Sigma_{\mathbf{s}}^{-1}$ the inverse matrix of $\Sigma_{\mathbf{s}}$ :

$$
\Sigma_{\mathbf{s}}^{-1}=\left(\begin{array}{ccc}
\varsigma_{1,1} & \varsigma_{1,2} & \cdots \\
\varsigma_{2,1} & \varsigma_{2,2} & \cdots \\
\vdots & & \ddots
\end{array}\right)
$$

Proposition 2.1. Assume we have a process defined as (8) with multivariate lognormal (MVLN) risk-neutral density with parameters (9), and the series is convergent. The IRND of the American option as defined in (1) will be

$$
f_{\mathbf{X}}=g_{\mathbf{S}}+\sum_{l_{1}=1}^{n}\left(M_{l_{1}}(-1)\left(-\frac{1}{S_{l_{1}}}+\frac{\partial \Lambda}{\partial S_{l_{1}}}\right) g_{\mathbf{S}}+\sum_{l_{2}=1}^{n} M_{l_{1}, l_{2}}\left(\frac{1}{2}\right) \frac{\partial^{2}}{\partial s_{l_{1}} \partial s_{l_{2}}} g_{\mathbf{S}}\right),
$$

where

$$
\begin{gathered}
\frac{\partial \Lambda}{\partial S_{l_{1}}}=-\frac{1}{S_{l_{1}}} \Sigma_{\mathbf{s},\left(l_{1},:\right)}^{-1}\left(\log (\mathbf{S})-\mu_{\mathbf{s}}\right) \\
\frac{\partial^{2}}{\partial s_{l_{1}} \partial s_{l_{2}}} g_{\mathbf{S}}=\left(\frac{1}{S_{l_{1}} S_{l_{2}}}-\frac{1}{S_{l_{1}}} \frac{\partial \Lambda}{\partial S_{l_{2}}}-\frac{1}{S_{l_{2}}} \frac{\partial \Lambda}{\partial S_{l_{1}}}+\frac{\partial \Lambda}{\partial S_{l_{1}}} \frac{\partial \Lambda}{\partial S_{l_{2}}}+\frac{\partial^{2} \Lambda}{\partial S_{l_{1}} \partial S_{l_{2}}}\right) g_{\mathbf{S}} \\
\frac{\partial^{2} \Lambda}{\partial S_{l_{1}}^{2}}=\frac{1}{S_{l_{1}}^{2}}\left(\Sigma_{\mathbf{s},\left(l_{1},:\right)}^{-1}\left(\log (\mathbf{S})-\mu_{\mathbf{s}}\right)-\varsigma_{l_{1}, l_{1}}\right) \\
\frac{\partial^{2} \Lambda}{\partial S_{l_{1}} \partial S_{l_{2}}}=-\frac{1}{S_{l_{1}} S_{l_{2}}} \varsigma_{l_{1}, l_{2}},
\end{gathered}
$$

and $\Sigma_{\mathbf{s},\left(l_{1},:\right)}^{-1}$ is the $l_{1}$ th row of $\Sigma_{\mathbf{s}}^{-1}$.

Proof. See Section A.1 of Appendix A.

For calculating $C_{E, 0, W,\left[l_{1}, \ldots, l_{j}\right]}(\Pi(\mathbf{X}(t)))$, the moments $M_{\left[l_{1},\left[\ldots,\left[l_{j}\right] \ldots\right]\right.}$ are given by the cumulants $k_{l_{1}, \ldots, l_{j}}(\mathbf{s})$ of the $\operatorname{MLVN}(\mu, \mathrm{S})$ distribution: 


$$
k_{l_{1}, \ldots, l_{j}}\left(g_{\mathbf{S}(t)}\right)=E\left[S_{1}^{\alpha_{1}} S_{2}^{\alpha_{2}} \ldots S_{n}^{\alpha_{n}}\right]=\exp \left(\frac{1}{2} \alpha^{\prime} \Sigma \alpha+\alpha^{\prime} \mu\right),
$$

where $\sum_{i} \alpha_{i}=j$, and the cumulants of the risk-neutral density of the price process $\mathbf{X}(t), k_{l_{1}, \ldots, l_{j}}(\mathbf{x})$. To calibrate the model, we will need to calculate $k_{l_{1}}\left(f_{\mathbf{X}(t)}\right), k_{l_{1}, l_{2}}\left(f_{\mathbf{X}(t)}\right)$, and $\lambda_{\mathrm{VCF}(2)}$. In Section 3, we provide a methodology for calculating the cumulants, and in Section 5 , we provide a calibration algorithm for finding $\lambda_{\mathrm{VCF}(2)}$.

Finally, using the results in Arismendi (2014), the analytical implied option price is

$$
\begin{aligned}
& C_{A, 0}(\Pi(\mathbf{S}(t)))=C_{E, 0}(\Pi(\mathbf{X}(t)))=C_{E, 0}(\Pi(\mathbf{S}(t)))+\sum_{j=1}^{2} C_{E, 0, W,\left[l_{1}, \ldots, l_{j}\right]}(\Pi(\mathbf{S}(t))) \\
& =\exp (-r t)\left[\int_{0}^{(n)} \Pi(\mathbf{s}(t)) g_{\mathbf{s}(t)} d \mathbf{s}\right. \\
& +\sum_{l_{1}=1}^{n} M_{l_{1}}(-1) \exp \left(-\mu_{l_{1}}\right)\left(\left(\Sigma_{\mathbf{s},\left(l_{1},:\right)}^{-1} \mu_{\mathbf{s}}-1\right) \int_{0}^{(n)} \Pi(\mathbf{s}(t)) g_{\mathbf{s}} d \mathbf{s}\right. \\
& \left.+\sum_{j=1}^{n} \varsigma_{l_{1}, j}^{(n)} \int_{0}^{\infty} \log \left(S_{j}\right) \Pi(\mathbf{s}(t)) g_{\mathbf{s}} d \mathbf{s}\right) \\
& +\sum_{l_{1}=1}^{n} M_{l_{1}, l_{1}} \frac{1}{2} \exp \left(-2 \mu_{l_{1}}\right)\left(\left(2-3 \Sigma_{\mathbf{s},\left(l_{1},:\right)}^{-1} \mu_{\mathbf{s}}+\left(\Sigma_{\mathbf{s},\left(l_{1},:\right)}^{-1} \mu_{\mathbf{s}}\right)^{2}-\varsigma_{l_{1}, l_{1}}\right)^{(n)}\right. \\
& \int_{0}^{\infty} \Pi(\mathbf{s}(t)) g_{\mathbf{s}} d \mathbf{s} .+\left(3-2 \Sigma_{\mathbf{s},\left(l_{1},:\right)}^{-1} \mu_{\mathbf{s}}\right) \sum_{j=1}^{n}\left(\varsigma_{l_{1}, j}\right) \int_{0}^{(n)} \Pi\left(\mathbf{s}(t) \log \left(S_{j}\right) g_{\mathbf{s}} d \mathbf{s}\right. \\
& \left.+\sum_{j_{1}=1}^{n}\left(\varsigma_{l_{1}, j_{1}}\right)^{2} \int_{0}^{(n)} \Pi(\mathbf{s}(t)) \log \left(S_{j_{1}}\right)^{2} g_{\mathbf{s}} d \mathbf{s}\right) \\
& +\sum_{l_{1}=1}^{n} \sum_{l_{2}=1}^{n} M_{l_{1}, l_{2}} \frac{1}{2} \exp \left(-\mu_{l_{1}}-\mu_{l_{2}}\right) \\
& \times\left(\left(1-\Sigma_{\mathbf{s},\left(l_{1},:\right)}^{-1} \mu_{\mathbf{s}}-\Sigma_{\mathbf{s},\left(l_{2},:\right)}^{-1} \mu_{\mathbf{s}}+\Sigma_{\mathbf{s},\left(l_{1},:\right)}^{-1} \mu_{\mathbf{s}} \Sigma_{\mathbf{s},\left(l_{2},:\right)}^{-1} \mu_{\mathbf{s}}-\varsigma_{l_{1}, l_{2}}\right)^{(n)}\right. \\
& \int_{0}^{\infty} \Pi(\mathbf{s}(t)) g_{\mathbf{s}} d \mathbf{s} .+\sum_{j=1}^{n}\left(\varsigma_{j, l_{1}}\left(1-\Sigma_{\mathbf{s},\left(l_{2},:\right)}^{-1} \mu_{\mathbf{s}}\right)+\varsigma_{l_{2}, j}\left(1-\Sigma_{\mathbf{s},\left(l_{1},:\right)}^{-1} \mu_{\mathbf{s}}\right)\right)^{(n)} \\
& \int_{0}^{\infty} \Pi(\mathbf{s}(t)) \log \left(S_{j}\right) g_{\mathbf{s}} d \mathbf{s} .+\sum_{j_{1}=1}^{n} \sum_{j_{2}=1}^{n}\left(\varsigma_{l_{1}, j_{1}} \varsigma_{l_{2}, j_{2}}\right) \\
& \left.\left.\int_{0}^{(n)} \Pi(\mathbf{s}(t)) \log \left(S_{j_{1}}\right) \log \left(S_{j_{2}}\right) g_{\mathbf{s}} d \mathbf{s}\right)\right]
\end{aligned}
$$


The integral:

$$
\int_{0}^{(n)} \Pi(\mathbf{s}(t)) g_{\mathbf{s}(t)} d \mathbf{s},
$$

is obtained with the approximations of $\mathrm{Li}$ et al. (2010) and Alexander and Venkatramanan (2012). The integrals,

$$
\begin{gathered}
\int_{-\infty}^{(n)} \Pi(\mathbf{s}(t)) \log \left(S_{j}\right) g_{\mathbf{s}} d \mathbf{s}, \\
\int_{-\infty}^{(n)} \Pi(\mathbf{s}(t)) \log \left(S_{j_{1}}\right) \log \left(S_{j_{2}}\right) g_{\mathbf{s}} d \mathbf{s},
\end{gathered}
$$

contain log-contracts. In Section 4, we apply the theory of multivariate truncated moments to compute these integrals.

\section{Implied multivariate moments of the risk-neutral density of American options}

The MGEE methodology presented relies on the moments of the risk-neutral density of the option. American options can be priced if a risk-neutral density resulting from the optimal exercise policy is estimated. In the present section, we develop an approximation of the moments of the empirical multivariate risk-neutral density of American options implied by (1) and (2), employing the Longstaff and Schwartz (2001) leastsquares method (LSM). These are inserted into the MGEE risk-neutral density and option pricing model presented in (6) and (7).

\subsection{LSM for path-dependent options pricing}

In Longstaff and Schwartz (2001), a path-simulation method is proposed for valuing the early exercise right of American options. Let $C_{t_{i}}^{O}, i \in\{1, \ldots, N\}$ represent the value of continuing with the option at time $t_{i}, 0 \leq t_{i}<t$, and $F\left(t_{i}, t_{j}, t\right)$ represent the cash flows of the option from time $t_{j}$ up to the maturity $t$ with the information available at time $t_{i}$, applying an optimal execution strategy. The option can be exercised only at discrete times $t_{1}, \ldots, t_{N}$, where $t_{N}=t$. The value of the continuation is the expected value of the cash flows:

$$
C_{t_{i}}^{O}=\exp (-r t) \mathbb{E}_{t_{i}}^{Q}\left[\sum_{j=i+1}^{N} F\left(t_{i}, t_{j}, t\right) \mid \mathcal{F}_{t_{i}}\right],
$$

where the option value is

$$
C_{A, 0}=\max \left(C_{0}^{O}, \Pi(\mathbf{S}(0))\right)
$$

where $\Pi\left(\mathbf{S}\left(t_{i}\right)\right)$ is the payoff function with the values of the assets at time $t_{i}$. 
The principle behind Longstaff and Schwartz's approach is to approximate the function of the cash flows $F\left(t_{i}, t_{j}, t\right)$ with a linear combination of basis functions $\psi_{L_{2}}$, where $L_{2}$ is the quadratically integrable function space. Later, the option's continuation value $C_{t_{i}}^{O}$, which is the expected value of the cash flows, is calculated adjusting the basis functions, applying the LSM. Longstaff and Schwartz test a set of Laguerre polynomials, but finally they suggest Chebyshev, Legendre and Hermite polynomials and other basis functions as substitutes. This procedure is done backwards, as the value of $F\left(t_{i}, t_{j}, t\right)$ could be greater than $F\left(t_{i}, t_{j+1}, t\right)$ and then will be optimal to exercise at time $t_{j}$. Let $\tilde{F}\left(t_{i}, t_{j}, t\right)$ be a simulated cash flow, calculated with the basis functions $\psi_{L_{2}}$, the value of continuation at $t_{0}=0$ can be approximated as

$$
C_{0}^{O}=\exp (-r t) N_{P}^{-1} \sum_{i=1}^{N_{P}}\left[\sum_{j=1}^{N} \tilde{F}\left(0, t_{j}, t\right) \mid \mathcal{F}_{0}\right],
$$

where $N_{P}$ are the number of simulated paths. All the $N$ cash flows can be subsumed into one cash flow. Define $\tilde{F}(0, t)$ as the sum of all cash flows carried back to time $t_{0}$ from one simulation path $i$ :

$$
\tilde{F}(0, t)=\left[\sum_{j=1}^{N} \tilde{F}\left(0, t_{j}, t\right) \mid \mathcal{F}_{0}\right]
$$

Finally, the value of the continuation is estimated as

$$
C_{t_{i}}^{O}=\exp (-r t) N_{P}^{-1} \sum_{i=1}^{N_{P}} \tilde{F}_{i}(0, t) .
$$

\subsection{Risk-neutral density of American options}

The LSM of Longstaff and Schwartz generates simulated paths to represent the cash flows that will be on average the function $F\left(t_{i}, t_{j}, t\right)$. To price a European option, it is not the path of the process that is relevant but the final value. We can use the notation $\mathbf{S}(t)$ for the one-period price process at final time $t$, and $\tilde{\mathbf{S}}\left(t, j, g_{\mathbf{S}(t)}\right)$ for the sample paths generated from this process, where $j$ is the path, and $g_{\mathbf{S}(t)}$ is the risk-neutral density of the process at time $t$. If the generating risk-neutral process is known, the riskneutral density can be estimated through simulations. Using the notation of the previous section, let $D(\mathbf{a}, \mathbf{b})$ represent the closed hypercube of the values of vector $\mathbf{S}(t)$ such that $a_{1} \leq S_{1}(t) \leq b_{1}, \ldots, a_{n} \leq S_{n}(t) \leq b_{n}$. Generate $N_{P}$ simulated paths $\tilde{\mathbf{S}}\left(t, j, g_{\mathbf{S}(t)}\right)=\left(\tilde{S}_{1}(t, j), \ldots, \tilde{S}_{n}(t, j)\right), j \in\left\{1, \ldots, N_{p}\right\}$ from the price process $\mathbf{S}(t)$. Denote by $\eta_{N_{P}}(D(\mathbf{a}, \mathbf{b}))$ the function that calculates the number of simulated paths that are inside $D(\mathbf{a}, \mathbf{b})$ :

$$
\eta_{N_{P}}(D(\mathbf{a}, \mathbf{b}))=\sum_{j=1}^{N_{P}} 1_{\left(\tilde{S}_{1}(t, j), \ldots, \tilde{S}_{n}(t, j)\right) \in D} .
$$


The empirical cumulative distribution function $(c d f), G_{\mathbf{S}(t)}$, can be obtained by integrating over the sample paths:

$$
F_{N_{P}}(\xi)=N_{P}^{-1(n)} \int_{-\infty}^{\xi_{i}} \eta_{N_{P}}\left(D\left(-\infty, \xi_{i}\right)\right)
$$

Assume $F_{N_{P}}(\xi)$ converge in distribution to $G_{\mathbf{S}(t)}$ :

$$
\lim _{N_{P} \rightarrow \infty} F_{N_{P}}(\xi)=G_{\mathbf{S}(t)}
$$

then the price of a European option with payoff $\Pi(\mathbf{S}(t))$ can be approximated by simulating $N_{P}$ paths from $g_{\mathbf{S}(t)}$ denoted by $\tilde{\mathbf{S}}\left(t, i, g_{\mathbf{S}(t)}\right)$, where $i \in\left\{1, \ldots, N_{P}\right\}$ is the path index, and averaging the payoff at the present value:

$$
C_{E, 0}=\exp (-r t) \mathbb{E}_{0}^{g_{\mathbf{S}}(t)}\left[\Pi(\mathbf{S}(t)) \mid \mathcal{F}_{0}\right] \approx \exp (-r t) N_{P}^{-1} \sum_{i=1}^{N_{P}} \Pi\left(\tilde{\mathbf{S}}\left(t, i, g_{\mathbf{S}(t)}\right)\right)
$$

Define an American option over the price process $\mathbf{S}(t)$ with the payoff $\Pi(\mathbf{S}(t))$, its value will be equal to:

$$
C_{A, 0}=\max \left(C^{O}(0), \Pi(\mathbf{S}(0))\right),
$$

with $C_{0}^{O}$ being the continuation value. Suppose that there exists another risk-neutral density $f_{\mathbf{X}}(t)$ such that

$$
C_{A, 0}=\exp (-r t) \mathbb{E}_{0}^{f_{\mathbf{X}}(t)}\left[\Pi(\mathbf{X}(t)) \mid \mathcal{F}_{0}\right]
$$

Applying a reverse engineering process, estimating the density $f_{\mathbf{X}}(t)$ from (20) is a similar procedure to that of inferring the implied volatility in the Black and Scholes' (1973) formula from the market prices. We will denote $f_{\mathbf{X}(t)}$ as the implied American risk-neutral density.

Equating (19) with (20), we have

$$
\max \left(C_{0}^{O}, \Pi(\mathbf{S}(0))\right)=\exp (-r t) \mathbb{E}_{0}^{f_{\mathbf{X}}(t)}\left[\Pi(\mathbf{X}(t)) \mid \mathcal{F}_{0}\right]
$$

By construction, if $\Pi(\mathbf{S}(0)) \geq C^{O}(0), f_{\mathbf{X}(t)}$ will have only one value. If $\Pi(\mathbf{S}(0))<C^{O}(0)$, and using (17) and (18), $f_{\mathbf{X}(t)}$ can be extracted from the relationship:

$$
C_{0}^{O}=\exp (-r t) \mathbb{E}_{0}^{f_{\mathbf{x}}(t)}\left[\Pi(\mathbf{X}(t)) \mid \mathcal{F}_{0}\right]
$$

At this point, the process $\mathbf{X}(t)$ has not been specified. Assume the process $\mathbf{X}(t)$ is equal to the process $\mathbf{S}(t)$, except when the process is absorbed by the early exercise condition to a barrier. If the European option $C_{E, 0}$ over the process $\mathbf{S}(t)$ for $t=t_{A}$ is exercised, then the process $\mathbf{X}(t)$ for $t \geq t_{A}$ will grow at the risk-free rate. Denote by $\tilde{\mathbf{X}}(t)$ a simulated path of the price process $\mathbf{X}(t)$, 


$$
\begin{aligned}
& \exp (-r t) \mathbb{E}_{0}^{g_{\mathrm{s}(t)}}\left[\sum_{j=1}^{N} F\left(0, t_{j}, t\right) \mid \mathcal{F}_{0}\right] \approx \exp (-r t) N_{P}^{-1} \sum_{i=1}^{N_{P}} \Pi\left(\tilde{\mathbf{X}}\left(t, i, f_{\mathbf{X}(t)}\right)\right) \\
& \exp (-r t) N_{P}^{-1} \sum_{i=1}^{N_{P}} \tilde{F}_{i}(0, t) \approx \exp (-r t) N_{P}^{-1} \sum_{i=1}^{N_{P}} \Pi\left(\tilde{\mathbf{X}}\left(t, i, f_{\mathbf{X}(t)}\right)\right) .
\end{aligned}
$$

Each cash flow $\tilde{F}_{i}(0, t)$ is equated to the final payoff function for path $i$, and we have a formula from which we can simulate paths with the distribution of $f_{\mathbf{X}(t)}$ :

$$
\Pi\left(\tilde{\mathbf{X}}\left(t, i, f_{\mathbf{X}(t)}\right)\right)=\tilde{F}_{i}(0, t) .
$$

We propose a method to extract $\tilde{\mathbf{X}}\left(t, i, f_{\mathbf{X}(t)}\right)$ from (21) as follows:

Denote by $\chi_{t_{i}, i}$ a function that indicates if the option was exercised at time $t_{i}$ for path $i$, and $\tilde{\mathbf{S}}\left(t_{i}, i, g_{\mathbf{S}(t)}\right)$ denote the value of a simulated path from the price process $\mathbf{S}(t)$. The values of $\chi_{t_{i}, i}$ are extracted by the LSM of Longstaff and Schwartz. If the option was exercised at time $t_{i}$, we project the payoff at time $t_{i}$ to the final time $t$. Using the payoff projected, a discount factor $d_{f}$ for each asset component $S_{j}\left(t_{i}\right)$ at time $t_{i}$ is calculated. The final simulated path value is

$$
\tilde{\mathbf{X}}\left(t, i, f_{\mathbf{X}(t)}\right)=\tilde{\mathbf{S}}\left(t, i, g_{\mathbf{S}(t)}\right) d_{f}^{-1}
$$

For example, define the payoff of a basket put option:

$$
\Pi(\mathbf{S}(t))=\left(K-\sum_{j=1}^{n} S_{j}(t)\right)^{+}
$$

for $n$ assets. The value of the simulated path from $f_{\mathbf{X}(t)}$ is

$$
\tilde{\mathbf{X}}\left(t, i, f_{\mathbf{X}(t)}\right)=\frac{\tilde{\mathbf{S}}\left(t, i, g_{\mathbf{S}(t)}\right)}{\left(\sum_{j=1}^{n} S_{j}\left(t_{i}\right) /\left(K-\exp \left(r\left(t-t_{i}\right)\right) \Pi\left(\mathbf{S}\left(t_{i}\right)\right)\right)\right)} .
$$

The implied cumulants can be calculated from $\tilde{\mathbf{X}}\left(t, i, f_{\mathbf{X}(t)}\right)$ as

$$
k_{l_{1}}\left(f_{\mathbf{X}(t)}\right)=\mathbb{E}_{0}^{Q}\left(\tilde{\mathbf{X}}\left(t, i, f_{\mathbf{X}(t)}\right)\right),
$$

and

$$
k_{l_{1}, l_{1}}\left(f_{\mathbf{X}(t)}\right)=\mathbb{E}_{0}^{Q}\left(\tilde{\mathbf{X}}\left(t, i, f_{\mathbf{X}(t)}\right)^{2}\right)-k_{l_{1}}\left(f_{\mathbf{X}(t)}\right)^{2}
$$

This methodology will generate sample paths that will be artificially linearly correlated. Then, the correlations are set to be equal:

$$
k_{l_{1}, l_{2}}\left(f_{\mathbf{X}(t)}\right)=k_{l_{1}, l_{2}}\left(g_{\mathbf{S}(t)}\right) .
$$

In Figure $\mathrm{C} 1$ of Appendix $\mathrm{C}$, we plot an empirical IRND calculated with the resulting sample paths $\tilde{\mathbf{X}}\left(t, i, f_{\mathbf{X}(t)}\right)$ as explained in this section. We considered put basket options 
without dividends. In this example, we can see the effects of the early exercise property over the IRND of a put option with different strike prices: it will create an additional mode below the strike price, and it will be higher while the option is more deep in-themoney (ITM).

\section{Pricing log-contracts}

The analytical option price (13) requires analytical expressions for integrals (15) and (16). In the case of basket options, where the payoff is defined as

$$
\Pi(\mathbf{S}(t), \omega, K)=\left[\omega_{1} S_{1}(t)+\cdots+\omega_{n} S_{n}(t)-K\right]^{+},
$$

the integrals (15) and (16) will have complex analytical expressions. In this section, we use the theory of multivariate truncated moments to price the log-contracts in a stepwise fashion,

$$
\begin{aligned}
\Pi\left(S_{1}(t), K\right) & =\log \left(S_{1}(t)\right)\left[S_{1}(t)-K\right]^{+}, \\
\Pi\left(S_{1}(t), S_{2}(t), K\right) & =\log \left(S_{1}(t)\right)\left[S_{2}(t)-K\right]^{+}, \\
\Pi\left(S_{1}(t), S_{2}(t), K\right) & =\log \left(S_{1}(t)\right)\left[S_{1}(t)-S_{2}(t)\right]^{+},
\end{aligned}
$$

in Sections 4.2, and 4.3, respectively. These results are used in Section 4.4 to price the general log-contract,

$$
\Pi(\mathbf{S}(t), \omega, K)=\log \left(S_{i_{1}}(t)\right) \log \left(S_{i_{2}}(t)\right)\left[\omega_{i_{1}} S_{i_{1}}(t)+\cdots+\omega_{i_{n}} S_{i_{n}}(t)-K\right]^{+},
$$

for $i_{1}, \ldots, i_{n} \in\{1, \ldots, n\}$. The log-contracts are relevant in pricing moment swaps, such as variance swaps, as pointed out by Demeterfi et al. (1999), Schoutens (2005), and Carr and Lee (2007), among others. In Arismendi (2014), the relationship between logcontracts and the risk-neutral density is explored.

\subsection{Multivariate truncated moments for pricing multi-asset options}

The risk-neutral density of multi-asset options can be defined in two different, but equivalent, approaches: The first approach is to define the risk-neutral density as the convolution of the univariate densities of each asset. For example, in the case of a basket option for geometric Brownian motion (GBM) processes, the risk-neutral density could be defined as the sum of the lognormal densities of the assets. The option price is the expected value of the payoff function over this univariate density. A second approach is to define the risk-neutral density as the multivariate density of the assets, where the marginals are the univariate densities corresponding to each asset with some dependence function. Copula theory could be applied in this case. The price of the option will be the expected value of the payoff, and this will be a function of the multivariate truncated moments of the risk-neutral density. In the first approach, the risk-neutral density is generally unknown but can be represented by a univariate integral, while in the second approach, the risk-neutral density is known, but the integral is multivariate. The pricing of multi-asset options will be 
reduced to express the expected payoff function, as a function of the multivariate truncated moments of the risk-neutral density.

Define the MVN distribution function as

$$
\Phi_{n}\left(x_{i} ; \Sigma_{\mathbf{s}}\right)=\int_{x_{i}}^{(n)} \phi_{n}\left(s_{i} ; \Sigma_{\mathbf{s}}\right) d \mathbf{s}
$$

where $i \in\{1, \ldots, n\}$ and $\phi_{n}\left(s_{i} ; \Sigma_{\mathbf{s}}\right)$ is the MVN density function with mean vector $\mathbf{0}$ and covariance matrix $\Sigma_{\mathbf{s}}$.

The following proposition allows us to price any option whose payoff can be reexpressed as a function of multivariate truncated moments: plain vanilla, power, capped power options and log-contracts are examples of such contracts. Although there exist closed-form formulae for these options, a general formula has never been presented:

Proposition 4.1. Let $\mathbf{X}(t)=\left(X_{1}(t), \ldots, X_{n}(t)\right)$ be a random vector with lower truncated multivariate standard lognormal distribution with correlation matrix $\mathbf{R}$, with truncation points $K_{i}$, for $i=\{1, \ldots, n\}$ such that $0<K_{i}$. The moments of order $p$, with $p=\sum_{i=0}^{n} \alpha_{i}$, of the distribution $\mathbf{X}$ truncated at $K_{i}$ are

$$
\mathbb{E}_{0}^{Q}\left[X_{1}(t)^{\alpha_{1}} \ldots X_{n}(t)^{\alpha_{n}} \mid X_{i}(t) \geq K_{i}\right]=L^{-1} \exp \left(-\frac{1}{2} \alpha^{\prime} \mathbf{R} \alpha\right) \Phi_{n}\left(b_{i} ; \mathbf{R}\right),
$$

where $b_{i}=\log \left(K_{i}\right)-\sum_{j} \rho_{i, j} \alpha_{j}$ and $L=\Phi_{n}\left(\xi_{i} ; \mathbf{R}\right)$ with $\xi_{i}=\log \left(K_{i}\right)$. If $\alpha=\mathbf{0}$ then $L=1$. If $\mathbf{X}(t)$ has a lower truncated MVLN distribution with mean vector $\mu$ and covariance matrix $\mathrm{S}$, the moments of order $p$ of $\mathbf{X}(t)$ are

$$
\mathbb{E}_{0}^{Q}\left[X_{1}(t)^{\alpha_{1}} \ldots X_{n}(t)^{\alpha_{n}} \mid X_{i}(t) \geq K_{i}\right]=L^{-1} \exp \left(\frac{1}{2} \alpha^{\prime} \Sigma^{-1} \alpha+\mu^{\prime} \alpha\right) \Phi_{n}\left(b_{i} ; \mathbf{R}\right),
$$

where $b_{i}=\left(\log \left(K_{i}\right)-\mu_{i}-\sigma_{i,:} \alpha\right) / \sigma_{i, i}$, with $\sigma_{i,:}$ the $i$ th row of $\Sigma$ and $L=\Phi_{n}\left(\xi_{i} ; \mathbf{R}\right)$ with $\xi_{i}=\left(\log \left(K_{i}\right)-\mu_{i}\right) / \sigma_{i}$.

Proof. Application of Proposition 6.1 in Arismendi (2013) yields the result.

\subsection{Log-contracts: sensitivity of the risk-neutral density}

Define a performance contract as a contingent claim with the payoff of the log-return of the underlyings, multiplied by a call option over one asset:

$$
\Pi\left(S_{1}(t), \ldots, S_{n}(t), K\right)=\log \left(\frac{S_{1}(t)}{S_{2}(t)}\right) \ldots \log \left(\frac{S_{n-1}(t)}{S_{n}(t)}\right)\left[S_{i}(t)-K\right]^{+},
$$

for $i \in\{1, \ldots, n\}$. Neuberger (2012) defined a similar contract, $S_{i}(t) \log \left(S_{i}(t)\right)$, as the entropy contract. The contract in (25) can be considered as an option over the entropy. The entropy contract derivation in Neuberger (2012) is the result of the calculation of the implied Black and Scholes (1973) generalized variance. In Schoutens (2005), the performance contract is derived as well as part of the calculation of univariate moment options. For example, the payoff of the performance contract of the return $S_{1}(t) / S_{2}(t)$ times $S_{3}(t) / S_{4}(t)$ subject to the value of the asset $S_{1}(t)$ greater than $K$ is 


$$
\Pi\left(S_{1}(t), \ldots, S_{4}(t), K\right)=\log \left(\frac{S_{1}(t)}{S_{2}(t)}\right) \log \left(\frac{S_{3}(t)}{S_{4}(t)}\right)\left[S_{1}(t)-K\right]^{+} .
$$

This can be the payoff of a warrant that a corporate executive will receive, the reward of the ratio revenue versus the costs of two departments: $\log \left(S_{1}(t) / S_{2}(t)\right) \log \left(S_{3}(t) / S_{4}(t)\right)$, times the profit of a goal revenue over a minimum threshold $K{ }^{8}$

Applying the properties of the log-function, the contract in (25) can be expressed similarly as

$$
\Pi\left(S_{1}(t), \ldots, S_{n}(t), K\right)=\left(\sum_{i=1}^{N} \log \left(S_{1}(t)\right)^{\alpha_{1, i}} \log \left(S_{2}(t)\right)^{\alpha_{2, i}} \ldots \log \left(S_{n}(t)\right)^{\alpha_{n, i}}\right)\left[S_{i}(t)-K\right]^{+},
$$

with $\alpha_{1, i}$ the number of times the variable $S_{1}(t)$ is in the numerator, for that particular component of the sum, and $N$ the total components of the sum $N=2^{j}$, with $j$ the total number of ratios. When $\mathbf{S}(t)$ behaves as a GBM as in (8), the change of variable $Y_{i}(t)=\log \left(S_{i}(t)\right)$, or $S_{i}(t)=\exp \left(Y_{i}(t)\right)$, can be applied to the MVLN risk-neutral density, and the resulting density will be a MVN distribution. The payoff (26) could transformed into

$$
\begin{aligned}
\Pi\left(S_{1}(t), \ldots, S_{4}(t), K\right) & =\Pi\left(\exp \left(Y_{1}(t)\right), \ldots, \exp \left(Y_{4}(t)\right), K\right) \\
& =\left(Y_{1}(t)-Y_{2}(t)+Y_{3}(t)-Y_{4}(t)\right)\left[\exp \left(Y_{1}(t)\right)-K\right]^{+}
\end{aligned}
$$

But $\left[\exp \left(Y_{1}(t)\right)-K\right]^{+}=\max \left(\exp \left(Y_{1}(t)\right)-K, 0\right)$, then

$$
\Pi\left(S_{1}(t), \ldots, S_{4}(t), K\right)=\left(Y_{1}(t)-Y_{2}(t)+Y_{3}(t)-Y_{4}(t)\right)\left[\exp \left(Y_{1}(t)\right)-K\right]_{\left\{Y_{1}(t) \geq \log (K)\right\}} .
$$

Under the risk-neutral measure, the value of the option of the contract $\Pi$ $\left(S_{1}(t), \ldots, S_{4}(t), K\right)$ will be

$$
\begin{aligned}
C_{0}(\Pi(\cdot))= & \exp (-r t) \mathbb{P}^{Q}\left(Y_{1}(t) \geq \log (K)\right) \\
& \times \mathbb{E}_{0}^{Q}\left[\left(Y_{1}(t)-Y_{2}(t)+Y_{3}(t)-Y_{4}(t)\right)\left(e^{Y_{1}(t)}-K\right) \mid Y_{1}(t) \geq \log (K)\right] \\
= & \exp (-r t) \mathbb{P}^{Q}\left(Y_{1}(t) \geq \log (K)\right)\left(\mathbb{E}_{0}^{Q}\left[Y_{1}(t)\left(e^{Y_{1}(t)}-K\right) \mid Y_{1}(t) \geq \log (K)\right]\right. \\
& +\mathbb{E}_{0}^{Q}\left[Y_{3}(t)\left(e^{Y_{1}(t)}-K\right) \mid Y_{1}(t) \geq \log (K)\right]-\mathbb{E}_{0}^{Q}\left[Y_{2}(t)\left(e^{Y_{1}(t)}-K\right) \mid Y_{1}(t) \geq \log (K)\right] \\
& \left.-\mathbb{E}_{0}^{Q}\left[Y_{4}(t)\left(e^{Y_{1}(t)}-K\right) \mid Y_{1}(t) \geq \log (K)\right]\right) .
\end{aligned}
$$

Each of the expected values can be decomposed as

$$
\begin{aligned}
\mathbb{E}_{0}^{Q}\left[Y_{i}(t)\left(e^{Y_{1}(t)}-K\right) \mid Y_{1}(t) \geq \log (K)\right]= & \mathbb{E}_{0}^{Q}\left[Y_{i}(t) e^{Y_{1}(t)} \mid Y_{1}(t) \geq \log (K)\right] \\
& -K \mathbb{E}_{0}^{Q}\left[Y_{i}(t) \mid Y_{1}(t) \geq \log (K)\right]
\end{aligned}
$$

The second term of (27) is just the first moment of a MVN distribution truncated at $\log (K)$. Similarly, the first term of (27) can be transformed in such a way that the resulting term will be the first truncated moment of a MVN distribution. This result can be demonstrated in a general manner: 
Proposition 4.2. Let $\mathbf{S}(t)=\left(S_{1}(t), \ldots, S_{n}(t)\right)$ be a multivariate GBM process defined as in (8), with MVLN distribution and parameters $\mu_{\mathbf{s}}, \Sigma_{\mathrm{s}}$, the conditional expected value:

$$
\mathbb{E}_{0}^{Q}\left[\log \left(S_{1}(t)\right)^{\alpha_{1}} \ldots \log \left(S_{n}(t)\right)^{\alpha_{n}} S_{1}(t)^{\beta_{1}} \ldots S_{n}(t)^{\beta_{n}} \mid S_{j}(t) \geq K\right]
$$

where $j \in\{1, \ldots, n\}$ is equal to the lower truncated moment of a MVN process $\mathbf{Y}(t)=$ $\left(Y_{1}(t), \ldots, Y_{n}(t)\right)$ times a constant $A$ :

$$
A \cdot \mathbb{E}_{0}^{Q}\left[Y_{1}(t)^{\alpha_{1}} \ldots Y_{n}(t)^{\alpha_{n}} \mid Y_{j}(t) \geq \log (K)\right],
$$

where $\mathbf{Y}(t) \sim N\left(\mu_{\mathbf{s}}+\Sigma_{\mathbf{s}} \beta, \Sigma_{\mathbf{s}}\right)$.

Proof. See Appendix A.2.

The final step to price performance contracts is finding the multivariate truncated moments of a MVN variable. We define some notation: Let $\mathbf{Y}(t)$ have a MVN distribution. Denote by $\mu_{\mathbf{y}}=\left(\mu_{1}, \ldots, \mu_{n}\right)$ the mean vector and $\Sigma_{\mathbf{y}}$ the covariance matrix of the component density $\phi_{n}\left(Y_{i} ; \mu_{\mathbf{y}}, \Sigma_{\mathbf{y}}\right)$ :

$$
\Sigma_{\mathbf{y}}=\left(\begin{array}{ccc}
\sigma_{y ; 1,1} & \cdots & \sigma_{y ; 1, n} \\
\vdots & \ddots & \vdots \\
\sigma_{y ; n, 1} & \cdots & \sigma_{y ; n, n}
\end{array}\right) .
$$

Define the truncation points $0<K_{i}, i=\{1, \ldots, n\}$. The distribution of $\mathbf{y}$ truncated on $K_{i}$ will be defined as the lower truncated $M V N$. Let $L$ be the total probability of truncated density function $\phi, L=\Phi_{n}\left(K_{i} ; \mathrm{S}_{\mathrm{s}}\right)$. Define $\psi=$ $\left(\psi_{1}, \ldots, \psi_{n}\right)$ as the vector of real coefficients of the moment generating function (MGF). The MGF of $\mathbf{Y}(t)$ is

$$
\mathbb{E}[\exp (\mathbf{\Psi Y})]=L^{-1}(2 \pi)^{-n / 2}\left|\Sigma_{\mathbf{s}}\right|^{-1 / 2} \exp (T)^{(n)} \int_{K_{i}}^{\infty} \exp \left(-\frac{1}{2}(\mathbf{y}-\zeta)^{\prime} \Sigma_{\mathbf{s}}^{-\mathbf{1}}(\mathbf{y}-\zeta)\right) d y_{i}
$$

where $\zeta=\Sigma_{\mathbf{s}} \boldsymbol{\Psi}$ and $T=\frac{1}{2} \boldsymbol{\Psi}^{\prime} \Sigma_{\mathbf{s}} \boldsymbol{\Psi}, \boldsymbol{\Psi}$ and $\mathbf{y}-\zeta$ are column vectors. If we define $b_{i}=K_{i}-\zeta_{i}$, then (28) becomes

$$
\mathbb{E}[\exp (\Psi \mathbf{y})]=L^{-1} \exp (T) \Phi_{n}\left(b_{s} ; \Sigma_{\mathbf{s}}\right) .
$$

To obtain arbitrary-order moments, a change of variable is applied, and then partial derivatives of (29) with respect to $\Psi$ must be derived and evaluated at $\Psi=0$. This methodology was applied in Arismendi (2013) to derive the moments, and we mention the notation used for the results' presentation. The univariate marginal $F_{y ; h_{1}}\left(K_{h_{1}}\right)$ over the distribution of $\mathbf{Y}(t)$ is defined as

$$
F_{y ; h_{1}}\left(K_{h_{1}}\right)=\int_{K_{1}}^{\infty} \cdots \int_{K_{h_{1}-1}}^{\infty} \int_{K_{h_{1}+1}}^{\infty} \cdots \int_{K_{n}}^{\infty} \phi_{n}\left(y_{h_{1}}=K_{h_{1}} ; \Sigma_{\mathbf{y}}\right)
$$


and the bivariate marginal as

$$
\begin{aligned}
F_{y ; h_{1}, h_{2}}\left(K_{h_{1}}, K_{h_{2}}\right)= & \int_{K_{1}}^{\infty} \cdots \int_{K_{h_{1}-1}}^{\infty} \int_{K_{h_{1}+1}}^{\infty} \cdots \int_{K_{h_{2}-1}}^{\infty} \int_{K_{h_{2}+1}}^{\infty} \cdots \int_{K_{n}}^{\infty} \phi_{n} \\
& \left(y_{h_{1}}=K_{h_{1}}, y_{h_{2}}=K_{h_{2}} ; \Sigma_{\mathbf{y}}\right) .
\end{aligned}
$$

Define the derivative of the marginal:

$$
Q_{j}\left(\xi_{h_{1}}, \ldots, \xi_{h_{p}}\right)=\frac{\partial}{\partial \psi_{j}}\left(F_{y ; h_{1}, \ldots, h_{p}}\left(\xi_{h_{1}}, \ldots, \xi_{h_{p}}\right)\right) .
$$

With these definitions, we derive the truncated moments of the MVN distribution:

Proposition 4.3. Let $\mathbf{Y}(t)$ be a process with a MVN distribution with cdf as (24) for $Y_{i}(t) \geq K_{i}, i=1,2, \ldots, n$, where $K_{i}$ are the truncation points over $\mathbf{Y}(t)$, and $\mu_{\mathbf{y}}, \Sigma_{\mathbf{y}}$ the mean vector and covariance matrix of the component density $\phi_{n}\left(Y_{i} ; \mu_{\mathbf{y}}, \Sigma_{\mathbf{y}}\right)$. Define the indices $i_{1}, \ldots, i_{4} \in\{1, \ldots, n\}$. Using a new notation, we denote the lower truncated p-moment of $\mathbf{Y}(t)$ by

$$
E_{0}^{Q}\left[Y_{i_{1}}(t) \ldots Y_{i_{p}}(t) \mid Y_{i}(t) \geq K_{i}\right]=m_{p,\left\{i_{1}, \ldots, i_{p}\right\}}\left(\mathbf{y}, K_{i}\right)
$$

The first four moments of $y$ are

$$
\begin{aligned}
m_{1,\left\{i_{1}\right\}}\left(\mathbf{y}, K_{i}\right)= & L^{-1}\left(\mu_{i_{1}} \Phi_{n}\left(\xi_{i} ; \mathbf{R}\right)+\sum_{h_{1}=1}^{n} \sigma_{y ; h_{1}, i_{1}} F_{y ; h_{1}}\left(\xi_{h_{1}}\right)\right) \\
m_{2,\left\{i_{1}, i_{2}\right\}}\left(\mathbf{y}, K_{i}\right)= & L^{-1}\left(\left(\mu_{i_{1}} \mu_{i_{2}}+\sigma_{y ; i_{1}, i_{2}}\right) \Phi_{n}\left(\xi_{i} ; \mathbf{R}\right)+\sum_{h_{1}=1}^{n}\left(\mu_{i_{1}} \sigma_{y ; h_{1}, i_{2}}+\mu_{i_{2}} \sigma_{y ; h_{1}, i_{1}}\right) F_{y ; h_{1}}\left(\xi_{h_{1}}\right)\right. \\
& \left.+\sum_{h_{1}=1}^{n} \sigma_{y ; h_{1}, i_{1}} Q_{i_{2}}\left(\xi_{h_{1}}\right)\right) \\
m_{3,\left\{i_{1}, i_{2}, i_{3}\right\}}\left(\mathbf{y}, K_{i}\right)= & L^{-1}\left\{\left(\mu_{i_{1}} \mu_{i_{2}} \mu_{i_{3}}+\mu_{i_{1}} \sigma_{y ; i_{2}, i_{3}}+\mu_{i_{2}} \sigma_{y ; i_{1}, i_{3}}+\mu_{i_{3}} \sigma_{y ; i_{1}, i_{2}}\right)\right. \\
& \Phi_{n}\left(\xi_{s} ; \mathbf{R}\right) \sum_{h_{1}=1}^{n} \mu_{i_{3}} \sigma_{y ; h_{1}, i_{1}} Q_{i_{2}}\left(\xi_{h_{1}}\right)+\sum_{h_{1}=1}^{n} \mu_{i_{2}} \sigma_{y ; h_{1}, i_{1}} Q_{i_{3}}\left(\xi_{h_{1}}\right) \\
& +\sum_{h_{1}=1}^{n} \mu_{i_{1}} \sigma_{y ; h_{1}, i_{2}} Q_{i_{3}}\left(\xi_{h_{1}}\right)+\sum_{h_{1}=1}^{n} F_{y ; h_{1}}\left(\xi_{h_{1}}\right)\left(\left(\mu_{i_{1}} \mu_{i_{2}}+\sigma_{y ; i_{1}, i_{2}}\right) \sigma_{y ; h_{1}, i_{3}}\right. \\
& \left.+\left(\mu_{i_{1}} \mu_{i_{3}}+\sigma_{y ; i_{1}, i_{3}}\right) \sigma_{y ; h_{1}, i_{2}}+\left(\mu_{i_{2}} \mu_{i_{3}}+\sigma_{y ; i_{2}, i_{3}}\right) \sigma_{y ; h_{1}, i_{1}}\right) \\
& +\sum_{h_{1}=1}^{n} \sigma_{y ; h_{1}, i_{1}}\left(\frac{\partial U_{i_{2}}\left(\xi_{h_{1}}\right)}{\partial t_{i_{3}}} F_{y ; h_{1}}\left(\xi_{h_{1}}\right)+U_{i_{2}}\left(\xi_{h_{1}}\right) Q_{i_{3}}\left(\xi_{h_{1}}\right)\right. \\
& \left.+\sum_{h_{2} \neq h_{1}}^{n} c_{h_{1} . s}\left(i_{2}, h_{2}\right) Q_{i_{3}}\left(\xi_{h_{1}}, \xi_{h_{2}}\right)\right) \\
&
\end{aligned}
$$




$$
\begin{aligned}
& m_{4,\left\{i_{1}, i_{2}, i_{3}, i_{4}\right\}}\left(\mathbf{y}, K_{i}\right)=L^{-1}\left\{\sum _ { h _ { 1 } = 1 } ^ { n } \left(\left(\mu_{i_{3}} \mu_{i_{4}}+\sigma_{y ; i_{3}, i_{4}}\right) \sigma_{y ; h_{1}, i_{1}} Q_{i_{2}}\left(\xi_{h_{1}}\right)\right.\right. \\
& +\left(\mu_{i_{2}} \mu_{i_{4}}+\sigma_{y ; i_{2}, i_{4}}\right) \sigma_{y ; h_{1}, i_{1}} Q_{i_{3}}\left(\xi_{h_{1}}\right)+\left(\mu_{i_{2}} \mu_{i_{3}}+\sigma_{y ; i_{2}, i_{3}}\right) \sigma_{y ; h_{1}, i_{1}} Q_{i_{4}}\left(\xi_{h_{1}}\right) \\
& +\left(\mu_{i_{1}} \mu_{i_{2}}+\sigma_{y ; i_{1}, i_{2}}\right) \sigma_{y ; h_{1}, i_{3}} Q_{i_{4}}\left(\xi_{h_{1}}\right)+\left(\mu_{i_{1}} \mu_{i_{3}}+\sigma_{y ; i_{1}, i_{3}}\right) \sigma_{y ; h_{1}, i_{2}} Q_{i_{4}}\left(\xi_{h_{1}}\right) \\
& \left.+\left(\mu_{i_{1}} \mu_{i_{4}}+\sigma_{y ; i_{1}, i_{4}}\right) \sigma_{y ; h_{1}, i_{2}} Q_{i_{3}}\left(\xi_{h_{1}}\right)\right)
\end{aligned}
$$

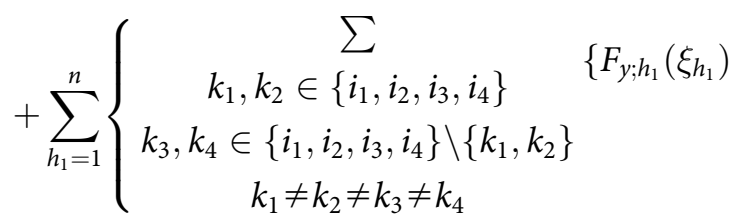

$$
\begin{aligned}
& \left(\mu_{k_{1}} \mu_{k_{2}} \mu_{k_{3}}+\mu_{k_{1}} \sigma_{y ; k_{2}, k_{3}}+\mu_{k_{2}} \sigma_{y ; k_{1}, k_{3}}+\mu_{k_{3}} \sigma_{y ; k_{1}, k_{2}}\right)\left(\sigma_{y ; h_{1}, k_{4}}\right) \\
& +\mu_{k_{1}} \sigma_{y ; h_{1}, k_{2}}\left(\frac{\partial U_{k_{3}}\left(\xi_{h_{1}}\right)}{\partial t_{k_{4}}} F_{y ; h_{1}}\left(\xi_{h_{1}}\right)+U_{k_{3}}\left(\xi_{h_{1}}\right) Q_{k_{4}}\left(\xi_{h_{1}}\right)\right. \\
& \left.+\sum_{h_{2} \neq h_{1}}^{n} c_{h_{1} . s}\left(k_{3}, k_{4}\right) Q_{k_{3}}\left(\xi_{h_{1}}, \xi_{h_{2}}\right)\right) \\
& +\sigma_{y ; h_{1}, k_{1}}\left(\frac{\partial U_{k_{2}}\left(\xi_{h_{1}}\right)}{\partial t_{k_{3}}} Q_{k_{4}}\left(\xi_{h_{1}}\right)+\frac{\partial U_{k_{2}}\left(\xi_{h_{1}}\right)}{\partial t_{k_{4}}} Q_{k_{3}}\left(\xi_{h_{1}}\right)\right. \\
& \left.\left.+U_{k_{2}}\left(\xi_{h_{1}}\right) \frac{\partial Q_{k_{3}}\left(\xi_{h_{1}}\right)}{\partial t_{k_{4}}}+\sum_{h_{2} \neq h_{1}} c_{h_{1} . s}\left(k_{2}, h_{2}\right) \frac{\partial Q_{k_{3}}\left(\xi_{h_{1}}\right)}{\partial t_{k_{4}}}\right)\right\} \\
& +\left(\mu_{i_{1}} \mu_{i_{2}} \mu_{i_{3}} \mu_{i_{4}}+\sigma_{y ; i_{1}, i_{2}} \sigma_{y ; i_{3}, i_{4}}+\sigma_{y ; i_{1}, i_{3}} \sigma_{y ; i_{2}, i_{4}}+\sigma_{y ; i_{1}, i_{4}} \sigma_{y ; i_{2}, i_{3}}\right. \\
& \left.\left.\sum_{\substack{k_{1}, k_{2} \in\left\{i_{1}, i_{2}, i_{3}, i_{4}\right\} \\
k_{3}, k_{4} \in\left\{i_{1}, i_{2}, i_{3}, i_{4}\right\} \backslash\left\{k_{1}, k_{2}\right\} \\
k_{1} \neq k_{2} \neq k_{3} \neq k_{4}}} \mu_{\left.k_{1} \mu_{k_{2}} \sigma_{y ; k_{3}, k_{4}}\right) \Phi_{n}(\xi)}\right\}\right\} .
\end{aligned}
$$

where

$$
\xi_{h_{1}}=\left(K_{h_{1}}-\mu_{h_{1}}\right) / \sigma_{h_{1}, h_{1}}^{2}
$$

Proof. This result follows from applying Proposition 4.1 of Arismendi (2013) with $\omega=1$.

To clarify the notation, the truncated moment of third-order $\mathbb{E}_{0}^{Q}\left[Y_{1}(t)^{2} Y_{2}(t)\right.$ $\left.\mid Y_{1}(t) \geq K\right]$ is equal to

$$
m_{3,\{1,1,2\}}\left(\mathbf{y}, K_{1}\right)
$$


Let $S_{1}(t)$ be a risk-neutral GBM process with constant volatility $\sigma$ as in (8). Using the results of Proposition 4.3, we price the contract $\Pi\left(S_{1}(t), K\right)=\log \left(S_{1}(t)\right)\left[S_{1}(t)-K\right]^{+}$, as

$$
\begin{aligned}
C_{0}\left(\Pi\left(S_{1}(t), K\right)\right) & =\exp (-r t) \mathbb{P}^{Q}\left(S_{1}(t) \geq K\right) \mathbb{E}_{0}^{Q}\left[\log \left(S_{1}(t)\right)\left(S_{1}(t)-K\right) \mid S_{1}(t) \geq K\right] \\
& =\exp (-r t) L_{1} \mathbb{E}_{0}^{Q}\left[Y_{1}(t)\left(e^{Y_{1}(t)}-K\right) \mid Y_{1}(t) \geq \log (K)\right] \\
& =\exp (-r t) L_{1}\left(\mathbb{E}_{0}^{Q}\left[Y_{1}(t) e^{Y_{1}(t)} \mid Y_{1}(t) \geq \log (K)\right]-K E_{0}^{Q}\left[Y_{1}(t) \mid Y_{1}(t) \geq \log (K)\right]\right) \\
& =\exp (-r t) L_{1}\left(\exp \left(\frac{1}{2} \beta^{2} \sigma^{2} t+\beta \mu_{s ; 1}\right) m_{1,\left\{i_{1}=1\right\}}(\mathbf{z}, K) \frac{L_{2}}{L_{1}}-K \cdot m_{1,\left\{i_{1}=1\right\}}(\mathbf{y}, K)\right)
\end{aligned}
$$

where $L_{1}=\mathbb{P}^{Q}\left(S_{1}(t) \geq K\right), L_{2}=\mathbb{P}^{Q}\left(Z_{1}(t) \geq \log (K)\right), \beta=1$ and

$$
\begin{aligned}
& m_{1,\left\{i_{1}=1\right\}}(\mathbf{y}, K)=L^{-1}\left(\mu_{y, 1} \Phi_{1}\left(\xi_{y ; 1} ; \mathbf{R}\right)+\sigma_{y ; 1,1} F_{y ; 1}\left(\xi_{y ; 1}\right)\right), \\
& m_{1,\left\{i_{1}=1\right\}}(\mathbf{z}, K)=L_{2}^{-1}\left(\mu_{z, 1} \Phi_{1}\left(\xi_{z ; 1} ; \mathbf{R}\right)+\sigma_{z ; 1,1} F_{z ; 1}\left(\xi_{z ; 1}\right)\right),
\end{aligned}
$$

with

$$
\begin{aligned}
& \xi_{y ; 1}=\frac{\left(K-\mu_{y ; 1}\right)}{\sigma_{y ; 1}^{2}}, \\
& \xi_{z ; 1}=\frac{\left(K-\mu_{z ; 1}\right)}{\sigma_{z ; 1}^{2}}, \\
& \mu_{y ; 1}=\mu_{z ; 1}=\mu_{s ; 1}=\log \left(S_{1}(0)\right)+\left(r-\frac{1}{2} \sigma^{2}\right) t, \\
& \sigma_{y ; 1}=\sigma_{z ; 1}=\sigma,
\end{aligned}
$$

and $Y(t) \sim N\left(\mu_{y ; 1}, \sigma_{y ; 1}^{2} t\right), Z(t) \sim N\left(\mu_{z ; 1}+\sigma_{y ; 1}^{2} t, \sigma_{z ; 1}^{2} t\right)$.

The results of Proposition 4.3 are produced not only for the univariate case but also for the multivariate. We price the bivariate contract $\Pi\left(S_{1}(t), S_{2}(t), K\right)=$ $\log \left(S_{1}(t)\right)\left[S_{2}(t)-K\right]^{+}$:

$$
\begin{aligned}
C_{0}\left(\Pi\left(S_{1}(t), S_{2}(t), K\right)\right) & =\exp (-r t) \mathbb{P}^{Q}\left(S_{2}(t) \geq K\right) \mathbb{E}_{0}^{Q}\left[\log \left(S_{1}(t)\right)\left(S_{2}(t)-K\right) \mid S_{2}(t) \geq K\right] \\
& =\exp (-r t) L_{1} \mathbb{E}_{0}^{Q}\left[Y_{1}(t)\left(e^{Y_{2}(t)}-K\right) \mid Y_{2}(t) \geq \log (K)\right] \\
& =\exp (-r t) L_{1}\left(\mathbb{E}_{0}^{Q}\left[Y_{1}(t) e^{Y_{2}(t)} \mid Y_{2}(t) \geq \log (K)\right]-K \mathbb{E}_{0}^{Q}\left[Y_{1}(t) \mid Y_{2}(t) \geq \log (K)\right]\right) \\
& =\exp (-r t) L_{1}\left(\exp \left(\frac{1}{2} \beta^{\prime} \Sigma_{\mathbf{s}} \beta+\beta^{\prime} \mu_{\mathbf{s}}\right) m_{1,\left\{i_{1}=1\right\}}(\mathbf{z}, K) \frac{L_{2}}{L_{1}}-K \cdot m_{1,\left\{i_{1}=1\right\}}(\mathbf{y}, K)\right),
\end{aligned}
$$

where $L_{1}=\mathbb{P}^{Q}\left(S_{2}(t) \geq K\right), L_{2}=\mathbb{P}^{Q}\left(Z_{2}(t) \geq \log (K)\right), \beta=(0,1)$ and

$$
\begin{aligned}
& m_{1,\left\{i_{1}=1\right\}}(\mathbf{y}, K)=L_{1}^{-1}\left(\mu_{y, 1} \Phi_{n}\left(\xi_{y ; i} ; \mathbf{R}\right)+\sum_{h_{1}=1}^{n=2} \sigma_{y ; h_{1}, 1} F_{y ; 1}\left(\xi_{y ; 1}\right)\right), \\
& m_{1,\left\{i_{1}=1\right\}}(\mathbf{z}, K)=L_{2}^{-1}\left(\mu_{z, 1} \Phi_{n}\left(\xi_{z ; i} ; \mathbf{R}\right)+\sum_{h_{1}=1}^{n=2} \sigma_{z ; h_{1}, 1} F_{z ; 1}\left(\xi_{z ; 1}\right)\right),
\end{aligned}
$$

with 


$$
\begin{aligned}
& \xi_{y ; i}=\frac{\left(K-\mu_{y ; i}\right)}{\sigma_{y ; i, i}^{2}}, \\
& \xi_{z ; i}=\frac{\left(K-\mu_{z ; i}\right)}{\sigma_{z ; i, i}^{2}},
\end{aligned}
$$

and $Y(t) \sim N\left(\mu_{\mathbf{s}}, \Sigma_{\mathbf{s}}\right), Z(t) \sim N\left(\mu_{\mathbf{s}}+\Sigma_{\mathbf{s}} \beta, \Sigma_{\mathbf{s}}\right)$

\subsection{Exchange option with log-contract (performance contract with a variable threshold)}

In all previous examples, the payoff contract has a fixed threshold $K$ over which the option is ITM. It could be the case that in the warrant of (26), the executive's payment is triggered only if the performance of an asset is over a variable threshold, rather than a fixed one. For example, a reward performance measure could be the revenue $S_{1}(t)$ over the cost of sales $S_{2}(t)$ times their ratio return:

$$
\Pi\left(S_{1}(t), S_{2}(t)\right)=\log \left(\frac{S_{1}(t)}{S_{2}(t)}\right)\left[S_{1}(t)-S_{2}(t)\right]^{+} .
$$

An option with this payoff function can be calculated using multivariate truncated moments' theory. The pricing function can be derived by calculating the expected moment with plane truncation as follows:

Proposition 4.4. Let $\mathbf{S}(t)=\left(S_{1}(t), \ldots, S_{n}(t)\right)$ be a multivariate GBM process defined as in (8), with MVLN distribution and parameters $\mu_{\mathbf{s}}, \Sigma_{\mathbf{s}}$, the conditional expected value:

$$
\mathbb{E}_{0}^{Q}\left[\log \left(S_{1}(t)\right)^{\alpha_{1}} \ldots \log \left(S_{n}(t)\right)^{\alpha_{n}} S_{1}(t)^{\beta_{1}} \ldots S_{n}(t)^{\beta_{n}} \mid S_{j}(t) \geq S_{i}(t)\right]
$$

where $i, j \in\{1, \ldots, n\}, j \neq i$, is equal to the lower truncated moment of a MVN process $\mathbf{Y}(t)=\left(Y_{1}(t), \ldots, Y_{n}(t)\right)$ times a constant $A$ :

$$
A \cdot \mathbb{E}_{0}^{Q}\left[Y_{1}(t)^{\alpha_{1}} \ldots Y_{n}(t)^{\alpha_{n}} \mid Y_{j}(t) \geq Y_{i}(t)\right]
$$

where $\mathbf{Y}(t) \sim N\left(\mu_{\mathbf{s}}+\Sigma_{\mathbf{s}} \beta, \Sigma_{\mathbf{s}}\right)$. Moreover, define a plane $B_{\mathbf{Y}(t)}=\left\{\mathbf{b}^{\prime} \mathbf{Y}(t) \geq p_{1}\right\}$, with $p_{1} \in \mathbb{R}, \mathbf{b} \in \mathbb{R}^{+}$, the first two moments,

$$
\begin{aligned}
\mathbb{E}_{0}^{Q}\left[Y_{i_{1}}(t) \mid B_{\mathbf{Y}(t)}\right] & =m_{1,\left\{i_{1}\right\}}\left(\mathbf{y}, B_{\mathbf{Y}(t)}\right), \\
\mathbb{E}_{0}^{Q}\left[Y_{i_{1}}(t) Y_{i_{2}}(t) \mid B_{\mathbf{Y}(t)}\right] & =m_{2,\left\{i_{1}, i_{2}\right\}}\left(\mathbf{y}, B_{\mathbf{Y}(t)}\right),
\end{aligned}
$$

of the variable $\mathbf{Y}(t)$ truncated on the plane $B_{\mathbf{Y}(t)}$ are

$$
\begin{aligned}
m_{1,\left\{i_{1}\right\}}\left(\mathbf{y}, B_{\mathbf{Y}(t)}\right) & =(L \gamma)^{-1} \phi_{1}\left(p_{2} / \gamma\right) \Sigma_{\mathbf{s}}\left(\mathbf{b}^{\prime} \mathbf{D}\right)+\mu_{\mathbf{s}}+\Sigma_{\mathbf{s}} \beta, \\
m_{2,\left\{i_{1}, i_{2}\right\}}\left(\mathbf{y}, B_{\mathbf{Y}(t)}\right) & =\Sigma_{\mathbf{s}}+\Sigma_{\mathbf{s}}\left(\mathbf{b}^{\prime} \mathbf{D}\right)\left(\mathbf{b}^{\prime} \mathbf{D}\right) \Sigma_{\mathbf{s}}\left(L \gamma^{2}\right)^{-1} \phi_{1}\left(p_{2} / \gamma\right)\left(p_{2} / \gamma-\phi_{1}\left(p_{2} / \gamma\right) / L\right),
\end{aligned}
$$

where $\gamma=\left(\left(\mathbf{b}^{\prime} \mathbf{D}\right) \Sigma_{\mathbf{s}}\left(\mathbf{b}^{\prime} \mathbf{D}\right)\right)^{1 / 2}, p_{2}=p_{1}-\mathbf{b}^{\prime} \mu_{\mathbf{s}}$ and $L=\mathbb{P}^{Q}\left(Y_{j}(t) \geq Y_{i}(t)\right)$. 
Proof. See Appendix A.3.

We price the bivariate contract $\left.\Pi\left(S_{1}(t), S_{2}(t), K\right)\right)=\log \left(S_{1}(t)\right)\left[S_{1}(t)-S_{2}(t)\right]^{+}$:

$$
\begin{aligned}
C_{0}\left(\Pi\left(S_{1}(t), S_{2}(t), K\right)\right)= & \exp (-r t) \mathbb{P}^{Q}\left(S_{1}(t) \geq S_{2}(t)\right) \mathbb{E}_{0}^{Q}\left[\log \left(S_{1}(t)\right)\left(S_{1}(t)-S_{2}(t)\right) \mid S_{1}(t) \geq S_{2}(t)\right] \\
= & \exp (-r t) L \mathbb{E}_{0}^{Q}\left[Y_{1}(t)\left(e^{Y_{1}(t)}-e^{Y_{2}(t)}\right) \mid Y_{1}(t) \geq Y_{2}(t)\right] \\
= & \exp (-r t) L\left(\mathbb{E}_{0}^{Q}\left[Y_{1}(t) e^{Y_{1}(t)} \mid Y_{1}(t) \geq Y_{2}(t)\right]-\mathbb{E}_{0}^{Q}\left[Y_{1}(t) e^{Y_{2}(t)} \mid Y_{1}(t) \geq Y_{2}(t)\right]\right) \\
= & \exp (-r t) L\left(\exp \left(\frac{1}{2} \beta_{1}^{\prime} \Sigma_{\mathbf{s}} \beta_{1}+\beta_{1}^{\prime} \mu_{\mathbf{s}}\right) m_{1,\left\{i_{1}=1\right\}}\left(\mathbf{z}, B_{\mathbf{Z}(t)}\right) \frac{L_{1}}{L}\right. \\
& \left.-\exp \left(\frac{1}{2} \beta_{2}^{\prime} \Sigma_{\mathbf{s}} \beta_{2}+\beta_{2}^{\prime} \mu_{\mathbf{s}}\right) m_{1,\left\{i_{1}=1\right\}}\left(\mathbf{x}, B_{\mathbf{X}(t)}\right) \frac{L_{2}}{L}\right)
\end{aligned}
$$

where $\quad \mathbf{b}=(1,-1), \quad B_{\mathbf{Z}(t)}=\left\{\mathbf{b}^{\prime} \mathbf{z} \geq 0\right\}, \quad B_{\mathbf{X}(t)}=\left\{\mathbf{b}^{\prime} \mathbf{x} \geq 0\right\}, \quad L=\mathbb{P}^{Q}\left(S_{1}(t) \geq S_{2}(t)\right)$, $L_{1}=\mathbb{P}^{Q}\left(Z_{1}(t) \geq Z_{2}(t)\right), L_{2}=\mathbb{P}^{Q}\left(X_{1}(t) \geq X_{2}(t)\right)$ and

$$
\begin{aligned}
& m_{1,\left\{i_{1}=1\right\}}\left(\mathbf{z}, B_{\mathbf{Z}(t)}\right)=\left(L_{1} \gamma\right)^{-1} \phi_{1}\left(p_{z} / \gamma\right) \Sigma_{\mathbf{s}}\left(\mathbf{b}^{\prime} \mathbf{D}\right), \\
& m_{1,\left\{i_{1}=1\right\}}\left(\mathbf{x}, B_{\mathbf{X}(t)}\right)=\left(L_{2} \gamma\right)^{-1} \phi_{1}\left(p_{x} / \gamma\right) \Sigma_{\mathbf{s}}\left(\mathbf{b}^{\prime} \mathbf{D}\right),
\end{aligned}
$$

where $\gamma=\left(\left(\mathbf{b}^{\prime} \mathbf{D}\right) \Sigma_{\mathbf{s}}\left(\mathbf{b}^{\prime} \mathbf{D}\right)\right)^{1 / 2}, p_{z}=-\left(\mathbf{b}^{\prime} \mathbf{D}\right)^{\prime}\left(\mu_{\mathbf{s}}+\Sigma_{\mathbf{s}} \beta_{1}\right), p_{x}=-\left(\mathbf{b}^{\prime} \mathbf{D}\right)^{\prime}\left(\mu_{\mathbf{s}}+\Sigma_{\mathbf{s}} \beta_{2}\right)$ and

$$
\begin{aligned}
& Y(t) \sim N\left(\mu_{\mathbf{s}}, \Sigma_{\mathbf{s}}\right), \\
& Z(t) \sim N\left(\mu_{\mathbf{s}}+\Sigma_{\mathbf{s}} \beta_{1}, \Sigma_{\mathbf{s}}\right), \\
& X(t) \sim N\left(\mu_{\mathbf{s}}+\Sigma_{\mathbf{s}} \beta_{2}, \Sigma_{\mathbf{s}}\right),
\end{aligned}
$$

with $\beta_{1}=(0,1), \beta_{2}=(1,0)$.

\subsection{An analytical expression for log-contracts of basket options}

\subsubsection{Extended Kirk approximation for basket options}

The Kirk (1996) approximation for spread options is an extension of the Margrabe (1978) formula for exchange options. Li, Deng and Zhou (2010) extend this method to the multivariate case. They transform the multivariate problem into a bivariate one using a common technique of approximating an arithmetic average as a geometric average, applied for Asian option calculations. Then, the standard Kirk (1996) approximation is applied to the resulting bivariate problem. We examine the Li, Deng and Zhou (2010) methodology as we will use it to approximate integrals (14)-(16). Subsequently, we use multivariate truncated moments to price these contracts.

Let $\omega_{i}, i \in\{1, \ldots, n\}$ be the portfolio weights associated with a set of assets with price process $\mathbf{S}(t)$. The payoff of a basket option is defined in (23). The multivariate problem of pricing a basket option is reduced to a bivariate one as follows.

Define $U_{i}(t)=\omega_{i} S_{i}(t), \mathbf{U}(t)=\left\{U_{1}(t), \ldots, U_{n}(t)\right\}$. Divide the set $\mathbf{U}(t)$ into the positives $\left\{U_{i}(t) \mid \omega_{i} \geq 0\right\}$, and the negatives $\left\{U_{j}(t) \mid \omega_{j}<0\right\}$. Let $m$ be the number of positives $\omega_{i}, 0 \leq m \leq n$. Select these $m$ variables from $\mathbf{U}(t)$ and denote such variables with indices $\left\{l_{1}, \ldots, l_{m}\right\}$. The remaining variables will be indexed as $\left\{U_{l_{m+1}}(t), \ldots, U_{l_{n}}(t)\right\}$. The absolute value arithmetic average of positive and negative sets is 


$$
\begin{aligned}
\bar{U}_{1, \ldots, m}(t) & =\frac{1}{m} \sum_{j=l_{1}, \ldots, l_{m}} U_{j}(t), \\
\bar{U}_{m+1, \ldots, n}(t) & =\frac{1}{m} \sum_{j=l_{m+1}, \ldots, l_{n}}-U_{j}(t),
\end{aligned}
$$

respectively. The sum of the positive and negative variables is equal to their arithmetic average times $m$ :

$$
U_{1}(t)+\cdots+U_{m}(t)=m \cdot \bar{U}_{1, \ldots, m}(t)
$$

The arithmetic average is approximated by a geometric average, and we set this geometric average as the variables $Y_{1}(t)$ and $Y_{2}(t)$ :

$$
\begin{aligned}
& Y_{1}(t)=m \cdot \prod_{j=l_{1}, \ldots, l_{m}} U_{j}(t)^{1 / m} \approx \sum_{j=l_{1}, \ldots, l_{m}} U_{j}(t), \\
& Y_{2}(t)=m \cdot \prod_{j=l_{m+1}, \ldots, l_{n}}\left(-U_{j}(t)\right)^{1 / n-m} \approx \sum_{j=l_{m+1}, \ldots, l_{n}}-U_{j}(t) .
\end{aligned}
$$

Swapping $Y(t)$ by $\omega^{\prime} \mathbf{S}(t)$, the payoff of the basket option becomes

$$
\Pi(\mathbf{S}(t), K)=\left[Y_{1}(t)-Y_{2}(t)-K\right]^{+},
$$

and then the problem is solved as the spread options in Appendix B.2. As the analytical approximation of Li, Deng and Zhou (2010) is an extension of the Kirk option price for spread options, there will be small approximation errors in the calculation of the integrals that will be calibrated applying volatility correction factors (VCF) as in Alexander and Venkatramanan (2012).

The basket option price based on the analytical approximation of Li, Deng and Zhou (2010) will be denoted as

$$
C_{\mathrm{Li} 2010}(\Pi(\mathbf{S}(t)), K, \mathrm{VCF})
$$

\subsubsection{Extended compound exchange options approximation for basket options}

Alexander and Venkatramanan (2012) employ a decomposition of the basket option into pairs of exchange options of calls and puts, that is, compound exchange options (CEO), where the assets are options, and the options price process is approximated as a GBM. For this reason we call this method the extended CEO approach. In essence, it is a recursive method where the final price is just the sum of sub-baskets of CEO. Denote by $\mathbf{S}(t)$ the asset price process defined in (8). Denote by $\Pi(\mathbf{S}(t), K)$ the payoff function of the basket option as in (23). Denote by $C_{n}(\Pi(\mathbf{S}(t), K))$ the price of the basket option. Set the sum of the first $m$ elements of the payoff contract equal to $S_{f}(t)$, and the sum of the $n-m$ remaining terms and the strike price as $S_{g}(t)$ :

$$
\begin{aligned}
\Pi(\mathbf{S}(t), K) & =\left[\omega_{1} S_{1}(t)+\cdots+\omega_{n} S_{n}(t)-K\right]^{+} \\
& =\left[S_{f}(t)+S_{g}(t)\right]^{+} .
\end{aligned}
$$

A value for $m$ could be half of the vector dimension: $m=\lfloor n / 2\rfloor$. The payoff of the basket option can be decomposed into smaller terms: 


$$
\Pi(\mathbf{S}(t), K)=\left[S_{f}(t)+S_{g}(t)\right]^{+}=\left[S_{f}(t)^{+}-S_{g}(t)^{-}\right]^{+}+\left[S_{g}(t)^{+}-S_{f}(t)^{-}\right]^{+} .
$$

This decomposition is applied recursively, and the minor terms will be plain vanilla calls/puts or spread options. The final option value will depend on the asset price, the strike $K$, the vector of weights of the basket option $\omega$ and the time $t$. Denote by $\xi$ a parameter having values $\{+1,-1\}$ that will be determined by the sign of the strike, and the type of sub-basket, if it is a put or a call sub-basket.

The value of the option has the form

$$
C_{0}(\Pi(\mathbf{S}(t), K)) \approx H_{1} \prod_{i} \Phi\left(G_{i}\right)+\cdots+H_{N} \prod_{j} \Phi\left(G_{j}\right)
$$

where $H_{i}$ and $G_{i}$ are functions of $\omega, \mathbf{S}(t), K$ and $\xi$. Then, the multivariate integral that represents the option price of the $n$-variate lognormal process can be approximated with a univariate function of univariate integrals of the standard normal. As a result, the extended CEO method is an approximation of the sum of lognormal random variables by the product of these lognormal random variables adjusted by an average volatility defined as the VCF.

The analytical basket option price approximation of Alexander and Venkatramanan (2012) is denoted as

$$
C_{\mathrm{AV} 2012}(\Pi(\mathbf{S}(t)), K, \mathrm{VCF}) .
$$

The multivariate contract $\Pi(\mathbf{S}(t), K)=\log \left(S_{1}(t)\right) \log \left(S_{2}(t)\right)\left[\sum_{i=1}^{n} \omega_{i} S_{i}(t)-K\right]^{+}$is priced. In order to provide an analytic approximation in this case we need to apply the extended Kirk or the extended CEO methodology. In both cases, we will have an approximated value. However, the extended $\mathrm{CEO}$ allows us to calibrate the average volatility defined as the VCF.

We apply the extended Kirk first, and then we apply the uncalibrated extended CEO for comparison.

\subsubsection{Extended Kirk method for log-contracts}

In the case of the extended Kirk method, the following variables are defined (Li, Deng, and Zhou 2010):

$$
\begin{aligned}
& S_{A}(t)=\frac{\sum_{i \in I} \omega_{i} S_{i}(t)}{S_{B}(t)+K}, \\
& S_{B}(t)=-\sum_{j \in J} \omega_{j} S_{j}(t),
\end{aligned}
$$

where $I$ is the set of indices from $i \in\{1, \ldots, n\}$, such that $\omega_{i}>0$, with $|I|=N_{I}$, and $J$ is the set of indices from $j \in\{1, \ldots, n\}$, such that $\omega_{j}<0$, with $|J|=N_{J}$. This variable is distributed: $S_{A}(t) \sim N\left(\mu_{A}, \sigma_{A}^{2} t\right)$, with 


$$
\begin{aligned}
\mu_{A} & =\log \left(\frac{\sum_{i \in I} \omega_{i} S_{i}(0)}{-\sum_{j \in J} \omega_{j} S_{j}(0)+\exp (-r t) K}\right)+\frac{1}{2} \sigma_{A}^{2} t, \\
\sigma_{A}^{2} & =v_{0}^{2}+\left(v_{A} F\right)^{2}-2 \rho_{A} v_{0} v_{A} F \\
F & =\frac{-\sum_{j \in J} \omega_{j} S_{j}(0)}{-\sum_{j \in J} \omega_{j} S_{j}(0)+\exp (-r t) K}, \\
v_{0} & =\frac{1}{N_{I}} \sqrt{\sum_{i_{1} \in I} \sum_{i_{2} \in I} \sigma_{i_{1}} \sigma_{i_{2}} \rho_{i_{1}, i_{2}}}, \\
v_{A} & =\frac{1}{N_{J}} \sqrt{\sum_{j_{1} \in J} \sum_{j_{2} \in J} \sigma_{j_{1}} \sigma_{j_{2}} \rho_{j_{1}, j_{2}}}, \\
\rho_{A} & =\frac{1}{N_{J} v_{A}} \sum_{j_{1} \in J} \sigma_{j_{1}}\left(\frac{1}{N_{I} v_{0}} \sum_{i_{1} \in I} \rho_{j_{1}, i_{1}} \sigma_{i_{1}}\right) .
\end{aligned}
$$

The payoff contract can be written as

$$
\Pi(\mathbf{S}(t), K)=\log \left(S_{1}(t)\right) \log \left(S_{2}(t)\right)\left[\left(-\sum_{j \in J} \omega_{j} S_{j}(t)+K\right)\left(S_{A}(t)-1\right)\right]^{+} .
$$

Applying a change of numeraire procedure similar to the exchange option, the price of the option under the risk-neutral measure is equal to

$$
\begin{aligned}
C_{0}(\Pi(\mathbf{S}(t), K)) & =\exp (-r t) \mathbb{E}_{0}^{Q}\left[\log \left(S_{1}(t)\right) \log \left(S_{2}(t)\right)\left[\left(-\sum_{j \in J} \omega_{j} S_{j}(t)+K\right)\left(S_{A}(t)-1\right)\right]^{+}\right] \\
& =\exp (-r t) \mathbb{E}_{0}^{Q}\left[\log \left(S_{1}(t)\right) \log \left(S_{2}(t)\right)\left(-\sum_{j \in J} \omega_{j} S_{j}(0)+\exp (-r t) K\right)\left[S_{A}(t)-1\right]^{+}\right] .
\end{aligned}
$$

Then, we have a trivariate contract over the variables $\left(S_{1}(t), S_{2}(t), S_{A}(t)\right)$, where the truncation is over $S_{A}(t)$, and this contract could be solved with Proposition 4.2 of performance contracts:

$$
\begin{aligned}
C_{0}(\Pi(\mathbf{S}(t), K))= & \exp (-r t) \mathbb{E}_{0}^{Q}\left[\log \left(S_{1}(t)\right) \log \left(S_{2}(t)\right)\left(-\sum_{j \in J} \omega_{j} S_{j}(0)+\exp (-r t) K\right)\left[S_{A}(t)-1\right]^{+}\right] \\
= & \exp (-r t)\left(-\exp (r t) \sum_{j \in J} \omega_{j} S_{j}(0)+K\right) \mathbb{P}^{Q}\left(S_{A}(t) \geq K\right) \\
& \times \mathbb{E}_{0}^{Q}\left[\log \left(S_{1}(t)\right) \log \left(S_{2}(t)\right)\left(S_{A}(t)-1\right) \mid S_{A}(t) \geq 1\right] \\
= & \exp (-r t)\left(-\sum_{j \in J} \omega_{j} S_{j}(0)+\exp (-r t) K\right) L_{1} \mathbb{E}_{0}^{Q}\left[Y_{1}(t) Y_{2}(t)\left(e^{Y_{A}(t)}-1\right) \mid Y_{A}(t) \geq 0\right] \\
= & \exp (-r t) L_{1}\left(\mathbb{E}_{0}^{Q}\left[Y_{1}(t) Y_{2}(t) e^{Y_{A}(t)} \mid Y_{A}(t) \geq 0\right]-\mathbb{E}_{0}^{Q}\left[Y_{1}(t) Y_{2}(t) \mid Y_{A}(t) \geq 0\right]\right) .
\end{aligned}
$$


In this multivariate case, the truncated moments of the variables $Y_{i}(t)$ are of second order

$$
C_{0}(\Pi(\mathbf{S}(t), K))=\exp (-r t) L_{1}\left(m_{2,\left\{i_{1}=1, i_{2}=2\right\}}(\mathbf{z}, 0) \frac{L_{2}}{L_{1}}-m_{2,\left\{i_{1}=1, i_{2}=2\right\}}(\mathbf{y}, 0)\right),
$$

where $L_{1}=\mathbb{P}^{Q}\left(S_{A}(t) \geq K\right), L_{2}=\mathbb{P}^{Q}\left(Z_{A}(t) \geq \log (K)\right)$ and

$$
\begin{aligned}
m_{2,\left\{i_{1}=1, i_{2}=2\right\}}(\mathbf{y}, K)= & L_{1}^{-1}\left(\left(\mu_{y ; 1} \mu_{y ; 2}+\sigma_{y ; 1,2}\right) \Phi_{3}\left(\xi_{y ; i} ; \mathbf{R}\right)\right. \\
& \left.+\sum_{h_{1}=1}^{3}\left(\mu_{y ; 1} \sigma_{y ; h_{1}, 2}+\mu_{y ; 2} \sigma_{y ; h_{1}, 1}\right) F_{y ; h_{1}}\left(\xi_{y ; h_{1}}\right)+\sum_{h_{1}=1}^{3} \sigma_{y ; h_{1}, 1} Q_{2}\left(\xi_{y ; h_{1}}\right)\right), \\
m_{2,\left\{i_{1}=1, i_{2}=2\right\}}(\mathbf{z}, K)= & L_{2}^{-1}\left(\left(\mu_{z ; 1} \mu_{z ; 2}+\sigma_{y ; 1,2}\right) \Phi_{3}\left(\xi_{z ; i} ; \mathbf{R}\right)\right. \\
& \left.+\sum_{h_{1}=1}^{3}\left(\mu_{z ; 1} \sigma_{z ; h_{1}, 2}+\mu_{z ; 2} \sigma_{z ; h_{1}, 1}\right) F_{z ; h_{1}}\left(\xi_{z ; h_{1}}\right)+\sum_{h_{1}=1}^{3} \sigma_{z ; h_{1}, 1} Q_{2}\left(\xi_{z ; h_{1}}\right)\right),
\end{aligned}
$$

with,

$$
\begin{aligned}
& \xi_{y ; i}=\left(K-\mu_{y ; i}\right) / \sigma_{y ; i, i}^{2}, \\
& \xi_{z ; i}=\left(K-\mu_{z ; i}\right) / \sigma_{z ; i, i}^{2}
\end{aligned}
$$

and $Y(t) \sim N\left(\mu_{\mathbf{s}}, \Sigma_{\mathbf{s}}\right), Z(t) \sim N\left(\mu_{\mathbf{s}}+\Sigma_{\mathbf{s}} \beta, \Sigma_{\mathbf{s}}\right), \beta=(0,0,1), \mu_{\mathbf{s}}=\left(\mu_{1}, \mu_{2}, \mu_{A}\right)$ and

$$
\Sigma_{\mathbf{s}}=\left(\begin{array}{ccc}
\sigma_{1}^{2} t & \rho_{1,2} \sigma_{1} \sigma_{2} t & \rho_{1, A} \sigma_{1} \sigma_{A} t \\
\rho_{1,2} \sigma_{1} \sigma_{2} t & \sigma_{2}^{2} t & \rho_{2, A} \sigma_{2} \sigma_{A} t \\
\rho_{A, 1} \sigma_{1} \sigma_{A} t & \rho_{A, 2} \sigma_{2} \sigma_{A} t & \sigma_{A}^{2} t
\end{array}\right) .
$$

We must find the correlation between $S_{A}, S_{1}(t)$ and $S_{2}(t)$. Denote by $\rho_{1, A}=\rho_{A, 1}$ the correlation between $S_{A}(t), S_{1}(t)$, and by $\rho_{2, A}=\rho_{A, 2}$ the correlation between $S_{A}(t), S_{2}(t)$. These correlations are estimated:

$$
\rho_{1, A}=\frac{\mathbb{E}_{0}^{Q}\left[S_{1}(t) S_{A}(t)\right]-\mathbb{E}_{0}^{Q}\left[S_{1}(t)\right] \mathbb{E}_{0}^{Q}\left[S_{A}(t)\right]}{\sqrt{\mathbb{V}^{Q}\left[S_{1}(t)\right] \mathbb{V}^{Q}\left[S_{A}(t)\right]}},
$$

with

$$
\begin{aligned}
& \mathbb{V}^{Q}\left[S_{1}(t)\right]=\mathbb{E}_{0}^{Q}\left[S_{1}(t)^{2}\right]-\mathbb{E}_{0}^{Q}\left[S_{1}(t)\right]^{2}, \\
& \mathbb{V}_{0}^{Q}\left[S_{A}(t)\right]=\mathbb{E}_{0}^{Q}\left[S_{A}(t)^{2}\right]-\mathbb{E}_{0}^{Q}\left[S_{A}(t)\right]^{2},
\end{aligned}
$$

the variance of the variables. The expected value and the variance are calculated: 


$$
\begin{aligned}
\mathbb{E}_{0}^{Q}\left[S_{1}(t)\right] & =S_{1}(0) \exp (r t), \\
\mathbb{V}_{0}^{Q}\left[S_{1}(t)\right] & =\left(\exp \left(\sigma_{1}^{2} t\right)-1\right) \exp \left(2 \mu_{1}+\sigma_{1}^{2} t\right), \\
\mu_{1} & =\log \left(S_{1}(0)\right)+\left(r-1 / 2 \sigma_{1}^{2}\right) t
\end{aligned}
$$

The variable $S_{A}$ is not lognormal, then

$$
\begin{aligned}
\mathbb{E}_{0}^{Q}\left[S_{A}(t)\right] & =\mathbb{E}_{0}^{Q}\left[\frac{S_{1}(t)}{S_{B}(t)+K}+\cdots+\frac{S_{N_{I}}(t)}{S_{B}(t)+K}\right] \\
& =\mathbb{E}_{0}^{Q}\left[\frac{S_{1}(t)}{S_{B}(t)+K}\right]+\cdots+\mathbb{E}_{0}^{Q}\left[\frac{S_{N_{I}}(t)}{S_{B}(t)+K}\right] .
\end{aligned}
$$

The variable $S_{B}(t)+K$ is not lognormal. Therefore, the fraction $\frac{S_{i}(t)}{S_{B}(t)+K}, i \in$ $\left\{1, \ldots, N_{I}\right\}$ will not be lognormal. But we note that the sum of the denominator could be approximated by a geometric average, as in the technique of Asian options, $S_{B}(t)+K \approx 2 S_{B}(t)^{1 / 2} K^{1 / 2}$, and the resulting fraction with this geometric average approximation will be lognormal:

$$
\begin{aligned}
\mathbb{E}_{0}^{Q}\left[S_{A}(t)\right] \approx & \mathbb{E}_{0}^{Q}\left[\frac{S_{1}(t)}{\left(N_{J}+1\right) \prod_{j \in J} S_{j}(t)^{1 /\left(N_{J}+1\right)} K^{1 /\left(N_{J}+1\right)}}\right]+\cdots+\mathbb{E}_{0}^{Q}\left[\frac{S_{N_{I}}(t)}{\left(N_{J}+1\right) \prod_{j \in J} S_{j}(t)^{1 /\left(N_{J}+1\right)} K^{1 /\left(N_{J}+1\right)}}\right] \\
= & \frac{1}{\left(N_{J}+1\right) K^{1 /\left(N_{J}+1\right)}} \mathbb{E}_{0}^{Q}\left[S_{1}(t)^{1} S_{j_{1}}(t)^{-1 /\left(N_{J}+1\right)} \ldots S_{j_{N_{J}}}(t)^{-1 /\left(N_{J}+1\right)}\right] \\
& +\cdots+\frac{1}{\left(N_{J}+1\right) K^{1 /\left(N_{J}+1\right)}} \mathbb{E}_{0}^{Q}\left[S_{S_{I}}(t)^{1} S_{j_{1}}(t)^{-1 /\left(N_{J}+1\right)} \ldots S_{j_{N_{J}}}(t)^{-1 /\left(N_{J}+1\right)}\right] \\
= & \frac{1}{\left(N_{J}+1\right) K^{1 /\left(N_{J}+1\right)}}\left(\exp \left(\frac{1}{2} \alpha_{S_{1}}^{\prime} \Sigma_{\mathbf{S}} \alpha_{S_{1}}+\alpha^{\prime}{ }_{S_{1}} \mu_{\mathrm{S}}\right)+\cdots+\exp \left(\frac{1}{2} \alpha_{S_{N_{I}}} \Sigma_{\mathbf{S}} \alpha_{S_{N_{I}}}+\alpha^{\prime}{ }_{S_{N_{I}}} \mu_{\mathrm{S}}\right)\right),
\end{aligned}
$$

where $\Sigma_{\mathbf{S}}, \mu_{\mathbf{S}}$ are defined in (9), and

$$
\begin{aligned}
\alpha_{S_{1}}= & \left(\alpha_{1}=1, \alpha_{2}=0, \alpha_{3}=0, \ldots, \alpha_{j_{1}}=-1 /\left(N_{J}+1\right), \ldots, \alpha_{j_{N_{J}+1}}=-1 /\left(N_{J}+1\right)\right), \\
& \vdots \\
\alpha_{S_{N_{I}}}= & \left(\alpha_{1}=0, \ldots, \alpha_{N_{I}}=1, \ldots, \alpha_{j_{1}}=-1 /\left(N_{J}+1\right), \ldots, \alpha_{j_{N_{J}+1}}=-1 /\left(N_{J}+1\right)\right),
\end{aligned}
$$

as in (12). Second moments of $S_{A}(t)$ are derived with the same method:

$$
\begin{array}{r}
\mathbb{E}_{0}^{Q}\left[S_{A}(t)^{2}\right] \approx \mathbb{E}_{0}^{Q}\left[\frac{S_{1}(t)^{2}}{\left(N_{J}+1\right)^{2} \prod_{j \in J} S_{j}(t)^{2 /\left(N_{J}+1\right)} K^{2 /\left(N_{J}+1\right)}}\right] \\
+\mathbb{E}_{0}^{Q}\left[\frac{S_{1}(t) S_{2}(t)}{\left(N_{J}+1\right)^{2} \prod_{j \in J} S_{j}(t)^{2 /\left(N_{J}+1\right)} K^{2 /\left(N_{J}+1\right)}}\right] \\
+\cdots+\mathbb{E}_{0}^{Q}\left[\frac{S_{i_{1}}(t) S_{i_{2}}(t)}{\left(N_{J}+1\right)^{2} \prod_{j \in J} S_{j}(t)^{2 /\left(N_{J}+1\right)} K^{2 /\left(N_{J}+1\right)}}\right] \\
+\cdots+\mathbb{E}_{0}^{Q}\left[\frac{S_{N_{I}}(t)^{2}}{\left(N_{J}+1\right)^{2} \prod_{j \in J} S_{j}(t)^{2 /\left(N_{J}+1\right)} K^{2 /\left(N_{J}+1\right)}}\right],
\end{array}
$$


with $i_{1}, i_{2} \in I$, and these expected moments are calculated as in (32) with the moments of a MVLN distribution as in (12). Finally, the variance $V^{Q}\left[S_{A}(t)\right]$ is derived and this yields the correlation $\rho_{1, A}$. The correlation $\rho_{2, A}$ is derived with the same algorithm.

\subsubsection{Extended CEO method for log-contracts}

In this case, our solution to the appearance of $\log (\cdot)$ functions is to introduce the $\log \left(S_{i}(t)\right)$ terms into the payoff function. However, these terms are positive for $S_{i}(t)>1$ and negative for $S_{i}(t)<1$ (Alexander and Venkatramanan 2012). A partition is made to the integral:

$$
\begin{aligned}
C_{0}(\Pi(\mathbf{S}(t), K))= & \exp (-r t) \mathbb{E}_{0}^{Q}\left[\log \left(S_{1}(t)\right) \log \left(S_{2}(t)\right)\left[\omega_{1} S_{1}(t)+\cdots+\omega_{n} S_{n}(t)-K\right]^{+}\right] \\
= & \exp (-r t) \int_{0}^{(n)}\left[\sum_{i=1}^{n} \omega_{i} S_{i}(t)-K\right]^{+} \log \left(S_{1}(t)\right) \log \left(S_{2}(t)\right) g_{\mathbf{S}(t)} \mathrm{d} g_{\mathbf{S}(t)} \\
= & \exp (-r t)\left(\int_{0}^{(n-2)}\left(\int_{0}^{\infty} \int_{0}^{1}\left[\left(\sum_{i=1}^{n} \omega_{i} S_{i}(t)-K\right) \log \left(S_{1}\right) \log \left(S_{2}\right)\right]^{+}\right) g_{\mathbf{S}(t)} \mathrm{d} g_{\mathbf{S}(t)}\right. \\
& +{ }^{(n-2)} \int_{0}^{\infty}\left(\int_{1}^{\infty} \int_{1}^{\infty}\left[\left(\sum_{i=1}^{n} \omega_{i} S_{i}(t)-K\right) \log \left(S_{1}\right) \log \left(S_{2}\right)\right]^{+}\right) g_{\mathbf{S}(t)} \mathrm{d} g_{\mathbf{S}(t)} \\
& +{ }^{(n-2)} \int_{0}^{\infty}\left(\int_{0}^{1} \int_{1}^{\infty}(-1)\left[\left(\sum_{i=1}^{n} \omega_{i} S_{i}(t)-K\right) \log \left(S_{1}\right) \log \left(S_{2}\right)\right]^{+}\right) g_{\mathbf{S}(t)} \mathrm{d} g_{\mathbf{S}(t)} \\
& \left.+{ }^{(n-2)} \int_{0}^{\infty}\left(\int_{1}^{\infty} \int_{0}^{1}(-1)\left[\left(\sum_{i=1}^{n} \omega_{i} S_{i}(t)-K\right) \log \left(S_{1}\right) \log \left(S_{2}\right)\right]^{+}\right) g_{\mathbf{S}(t)} d g_{\mathbf{S}(t)}\right)
\end{aligned}
$$

These partitions will create $2^{N}$ additional integrals for each integral with a $\log \left(S_{i}(t)\right)$ term, where $N$ is the number of different logarithmic terms:

$$
={ }^{(n-1)} \int_{0}^{\infty} \int_{0}^{1} \int_{0}^{1} \int_{0}^{1} \int_{0}^{1}(\cdot)+{ }^{(n-1)} \int_{0}^{\infty} \int_{0}^{1} \int_{0}^{1} \int_{0}^{1} \int_{1}^{\infty}(\cdot)+\cdots+{ }^{(n-1)} \int_{0}^{\infty} \int_{1}^{\infty} \int_{1}^{\infty} \int_{1}^{\infty} \int_{1}^{\infty}(\cdot) .
$$

We have made a partition and we introduce the $\log (\cdot)$ functions into the payoff, the extended CEO method will recursively introduce these $\log (\cdot)$ functions into the smallest contract to value:

$$
\mathbb{E}_{0}^{Q}\left[\left[S_{i}(t)-K\right]^{+} \log \left(S_{1}(t)\right) \log \left(S_{2}(t)\right)\right]
$$

and this contract is solved with the results of Section 4.2 .

\section{Calibration}

In this section, we calibrate the higher order moments parameter $\lambda_{\mathrm{vcf}(2)}$, and the VCF defined in Section 4 for the integrals (14)-(16). In Section 2, the IRND (11) was presented, with the corresponding analytical option price (13). The parameters of the risk-neutral density are the cumulants $k_{l_{1}}\left(f_{\mathbf{X}(t)}\right)$ and $k_{l_{1}, l_{2}}\left(f_{\mathbf{X}(t)}\right)$, and the calibration parameter of higher order moments $\lambda_{\mathrm{vcf}(2)}$. The cumulants are calculated in Section 3 , 
applying a reverse engineering algorithm of the Longstaff and Schwartz (2001) method. In Section 4.4, an analytical expression for the option price of a basket option is developed, but as the risk-neutral density expansion (11) is sensitive to small errors, the analytical integrals (15) and (16) need to be calibrated as in Alexander and Venkatramanan (2012). We define three VCFs:

(1) $\mathbf{V C F}(\mathbf{W})$ for calibrating the integral $C_{E, 0}(\Pi(\mathbf{S}(t)))$,

(2) $\mathbf{V C F}(\mathbf{1})$ for calibrating integrals of first-order cumulants difference $C_{E, 0, W,\left[l_{1}\right]}(\Pi(\mathbf{S}(t)))$,

(3) $\mathbf{V C F}(2)$ for calibrating integrals of second-order cumulants difference $C_{E, 0, W,\left[l_{1}, l_{2}\right]}(\Pi(\mathbf{S}(t)))$.

As the $\operatorname{VCF}(2)$ affects the integrals of the second-order cumulants, we simplify the calibration algorithm by adjusting the higher order moment parameter,

$$
\lambda_{v c f(2)}=\operatorname{VCF}(2) \text {. }
$$

The parameter $\operatorname{VCF}(\mathrm{W})$ is calibrated finding the root of

$$
h_{\mathrm{VCF}(\mathrm{W})}=C_{-}(\Pi(\mathbf{S}(t)), K, \mathrm{VCF})-C_{E, 0}(\Pi(\mathbf{S}(t))),
$$

the parameter $\operatorname{VCF}(1)$ is calibrated finding the root of

$$
\begin{aligned}
h_{\mathrm{VCF}(1)}= & \exp (-r t) \sum_{l_{1}=1}^{n} M_{l_{1}}(-1)^{(n)} \int_{0}^{\infty} \Pi(\mathbf{S}(t)) \frac{\partial}{\partial S_{l_{1}}} g_{\mathbf{S}} \\
& -\sum_{l_{1}=1}^{n} M_{l_{1}}(-1) \exp \left(-\mu_{l_{1}}\right)\left(\left(\Sigma_{\mathbf{s},\left(l_{1},:\right)}^{-1} \mu_{\mathbf{s}}-1\right) C_{-}(\Pi(\mathbf{S}(t)), K, \mathrm{VCF})\right. \\
& \left.+\sum_{j=1}^{n} \varsigma_{l_{1}, j} C_{-}\left(\log \left(S_{j}\right) \Pi(\mathbf{S}(t)), K, \mathrm{VCF}\right)\right)
\end{aligned}
$$

and the parameters $\operatorname{VCF}(2)=\lambda_{\mathrm{VCF}(2)}$ are calibrated finding the root of

$$
h_{\mathrm{VCF}(2)}=C_{A, 0}(\Pi(\mathbf{S}(t)))-C_{E, 0}(\Pi(\mathbf{X}(t))),
$$

exchanging the integrals (14)-(16) by $C_{-}(\Pi(\mathbf{S}(t)), K, \mathrm{VCF}), \quad C_{-}\left(\log \left(S_{j}\right) \Pi(\mathbf{S}(t))\right.$, $K, \mathrm{VCF})$ and $C_{-}\left(\log \left(S_{i}\right) \log \left(S_{j}\right) \Pi(\mathbf{S}(t)), K, \mathrm{VCF}\right)$, respectively, where $C_{-}$is $C_{\mathrm{Li} 2010}$ $(\Pi(\mathbf{S}(t)), K, \mathrm{VCF})$ for the case of the Li, Deng and Zhou (2010) approximation, and $C_{\mathrm{AV} 2012}(\Pi(\mathbf{S}(t)), K, \mathrm{VCF})$ for the case of the Alexander and Venkatramanan (2012) approximation.

\subsection{Numerical example}

Define the multi-asset risk-neutral GBM price processes $\mathbf{S}(t)$ with the parameters $\sigma_{i}=0.2, \mathbf{S}(0)=\left(S_{1}(0), \ldots, S_{5}(0)\right)=(35,25,20,15,5), i \in\{1, \ldots, 5\}$. Define the payoff of a basket put option by 


$$
\Pi(\mathbf{S}(t))=\left(K-\sum_{i=1}^{5} S_{i}(t)\right)^{+}
$$

with $K \in\{90,100,110\}$. We calculate the IRND for different interest rates and expirations: $r \in\{0.05,0.10\}, t \in\{0.25,1\}$. Figure $\mathrm{C} 1$ of Appendix $\mathrm{C}$ exhibits the empirical density of the sum of asset prices $X_{i}(t)$, generated from the multi-asset GBM density $g_{\mathbf{S}(t)}$ and the empirical implied American risk-neutral densities $f_{\mathbf{X}(t)}$. The difference of the densities for different strikes shows that the early exercise condition of the American options is reflected by the appearance of an additional mode located in the ITM region of the option. This happens because the strike prices are below the expected asset prices:

$$
\mathbb{E}_{0}^{g_{\mathrm{S}(t)}}\left[\sum_{j=1}^{n} S_{j}(t)\right]=\sum_{j=1}^{n} S_{j}(0) \exp (r t)>K_{i}
$$

For ITM options, Figure $\mathrm{C} 1$ demonstrates that the density concentration around the second mode is proportionally smaller than the original first mode of the multivariate GBM process. On the contrary, for out-of-the-money (OTM) options, the distribution mode generated by the process of calculating $f_{\mathbf{X}(t)}$ from $g_{\mathbf{S}(t)}$ has a much larger mode than the original one. These characteristics in $f_{\mathbf{X}(t)}$ are the result of more sample paths exercising earlier for ITM options than for OTM options. Table C1 of Appendix C displays the results of the calibrated American IRND $f_{\mathbf{X}(t)}$ by the risk-neutral density $g_{\mathbf{S}(t)}$. We want to study the effect of the early exercise property on the moments of the risk-neutral density $g_{\mathbf{S}(t)}$. The column American is the option price of the Longstaff and Schwartz LSM. The prices when the option is calculated with the expected value of the implied density $f_{\mathbf{X}(t)}$ are identical by construction. The column European shows the European option price. The early exercise premium increases the value of the option. In Figure C1, the difference between the European risk-neutral density and the implied American risk-neutral density is plotted. The early exercise condition shifts the density from the OTM region towards the ITM region to compensate for the premium. Figures C2 and C3 show the IRND for a multivariate Merton (1976) jump-diffusion process. In the jump-diffusion case, the difference between the risk-neutral density and the American IRND is lower, due to the higher volatility consequence of the jumps.

\section{Conclusions}

Risk-neutral valuation is an important concept for the pricing of contingent claims. European options can be priced through the expected value of the payoff function when the risk-neutral density of the assets is available. However, for path-dependent options, this approach cannot be applied. In this paper, we develop a model-free multivariate riskneutral density under which the expected value of the payoff function is equal to the price of an American option. An analytical formula for the price of a basket option is also provided. The risk-neutral density is approximated with a semi-parametric method, the MGEE. The difference in risk-neutral density moments, needed for the MGEE, is derived 
from the early exercise premium, which is approximated with a reverse engineering approach based on the Longstaff and Schwartz LSM. The MGEE is carried up to the second-order moment, and the premium associated with higher order moments is included in the volatility through a calibration algorithm. Analytical methods are generally preferred when pricing options. However, numerical methods have generally to be used for pricing American options. The development of analytical formulae in option pricing is crucial for calculating Greeks and for a better understanding of model implications. We propose the theory of multivariate truncated moments as a central methodology to derive analytical formulae for the pricing of American multi-asset option contracts. A general result is derived to price options defined on assets that follow multivariate GBM processes and then is applied to price new exotics options defined on multi-asset log-contracts, defined as performance options. These multi-asset log-contracts represent the sensitivities of the price of the option to changes in the moments of the risk-neutral density, and they were used in moment swap valuation. The theory of multivariate truncated moments gains importance when the price of options is computed with the multivariate density of the assets, instead of the univariate density of the payoff function. Truncation has been an active topic of research in statistics and mathematics for more than five decades, and all these results could be used for pricing American and European multi-asset options as well. Finally, a calibration algorithm is provided, for fitting the VCF to value basket options, as proposed by Alexander and Venkatramanan (2012). The same approach is used for calibrating the Li, Deng and Zhou (2010) formula. The resulting IRND includes the effects of the early exercise premium, producing a shifting of the risk-neutral density towards the ITM region. Potential extensions of our work include producing an implied European riskneutral density, given American prices in the market. This could be considered as a multivariate extension of Tian (2011). Furthermore, one could derive similar implied risk-neutral densities for other more general and complex path-dependent conditions.

\section{Notes}

1. Broadie and Detemple (1997) developed a set of results related to the exercise region for multi-asset options.

2. Rubinstein (1998) provides an extension of the implied Binomial tree for the cases when skewness and excess kurtosis are different to zero.

3. The payoff of an exchange option is $\Pi(\mathbf{S}(t))=\left[S_{1}(t)-S_{2}(t)\right]+$, with $\mathbf{S}(t)$ being a bivariate asset price process.

4. Approximations based on arithmetic Brownian motions are presented by Poitras (1998), applying the theory developed by Goldenberg (1991). Similarly, Shimko (1994) applies Goldenberg's results on futures markets.

5. See also Haug (2006) for an extensive list of analytic formulae for option pricing.

6. In Filipović et al. (2013), a profound study of transition probabilities of affine jumpdiffusions is presented that could serve as mathematical support for the polynomial expansions of distributions.

7. Models with stochastic volatility could be approximated by adjusting moments $M_{l_{1}, l_{2}}$.

8. It is important to highlight that, although the truncation or the positive part function of the definition of the performance contract we are providing here is applied only over one variable, truncation over several variables can be solved with the results we are presenting. A more complex case, where the truncation is over the sum of several variables, is presented in the multivariate performance contract section. 


\section{Disclosure statement}

No potential conflict of interest was reported by the authors.

\section{ORCID}

J. C. Arismendi (D) http://orcid.org/0000-0002-3163-5434

\section{References}

Alexander, C., and A. Venkatramanan. 2012. "Analytic Approximations for Multi-Asset Option Pricing.” Mathematical Finance 22 (4): 667-689. doi:10.1111/mafi.2012.22.issue-4.

Arismendi, J. C. 2013. "Multivariate Truncated Moments." Journal of Multivariate Analysis 117: 41-75. doi:10.1016/j.jmva.2013.01.007.

Arismendi, J. C. 2014. "A Multi-Asset Option Approximation for General Stochastic Processes." SSRN Electronic Journal. doi:10.2139/ssrn.2428216.

Barone-Adesi, G., and R. E. Whaley. 1987. "Efficient Analytic Approximation of American Option Values." The Journal of Finance 42 (2): 301-320. doi:10.1111/j.1540-6261.1987.tb02569.x.

Black, F., and M. Scholes. 1973. "The Pricing of Options and Corporate Liabilities." Journal of Political Economy 81 (3): 637-654. doi:10.1086/260062.

Borovkova, S., F. J. Permana, and H. V. D. Weide. 2007. "A Closed Form Approach to the Valuation and Hedging of Basket and Spread Options." The Journal of Derivatives 14 (4): 824. doi:10.3905/jod.2007.686420.

Breeden, D. T., and R. H. Litzenberger. 1978. "Prices of State-Contingent Claims Implicit in Option Prices." The Journal of Business 51 (4): 621-651. doi:10.1086/jb.1978.51.issue-4.

Broadie, M., and J. Detemple. 1997. "The Valuation of American Options on Multiple Assets." Mathematical Finance 7 (3): 241-286. doi:10.1111/mafi.1997.7.issue-3.

Carr, P. 1988. "The Valuation of Sequential Exchange Opportunities', the Journal of Finance 43 (5),1235-1256. Carr, P. and R. Lee (2007), 'Realised Volatility and Variance: Options via Swaps.” RISK 20 (5): 76-83.

Carr, P., and R. Lee. 2007. "Realised Volatility and Variance: Options Via Swaps.” Risk 20 (5): 7683.

Corrado, C. J., and T. Su. 1996. "SP 500 Index Option Tests of Jarrow and Rudd's Approximate Option Valuation Formula." Journal of Futures Markets 16 (6): 611-629. doi:10.1002/(ISSN) 1096-9934.

Demeterfi, K., E. Derman, M. Kamal, and J. Zou. 1999. More than You Ever Wanted to Know about Volatility Swaps. Technical report. New York: Goldman Sachs \& Co.

Filipović, D., E. Mayerhofer, and P. Schneider. 2013. "Density Approximations for Multivariate Affine Jump-Diffusion Processes.” Journal of Econometrics 176 (2): 93-111. doi:10.1016/j. jeconom.2012.12.003.

Flamouris, D., and D. Giamouridis. 2002. "Estimating Implied Pdfs from American Options on Futures: A New Semipara- Metric Approach.” Journal of Futures Markets 22 (1): 1-30. doi:10.1002/fut.2205.

Goldenberg, D. H. 1991. “A Unified Method for Pricing Options on Diffusion Processes.” Journal of Financial Economics 29 (1): 3-34. doi:10.1016/0304-405X(91)90011-8.

Haug, E. G. 2006. The Complete Guide to Option Pricing Formulas. New York: McGraw-Hill.

Jackwerth, J. C., and M. Rubinstein. 1996. "Recovering Probability Distributions from Option Prices." The Journal of Finance 51 (5): 1611-1631. doi:10.1111/j.1540-6261.1996.tb05219.x.

Jarrow, R., and A. Rudd. 1982. "Approximate Option Valuation for Arbitrary Stochastic Processes." Journal of Financial Economics 10 (3): 347-369. doi:10.1016/0304-405X(82)90007-1.

Jondeau, E., and M. Rockinger. 2000. "Reading the Smile: The Message Conveyed by Methods Which Infer Risk Neutral Densities." Journal of International Money and Finance 19 (6): 885915. doi:10.1016/S0261-5606(00)00036-X. 
Jondeau, E., and M. Rockinger. 2001. "Gram-Charlier Densities." Journal of Economic Dynamics and Control 25 (10): 1457-1483. doi:10.1016/S0165-1889(99)00082-2.

Ju, N. 1998. "Pricing by American Option by Approximating Its Early Exercise Boundary as a Multipiece Exponential Function.” Review of Financial Studies 11 (3): 627-646. doi:10.1093/ $\mathrm{rfs} / 11.3 .627$.

Ju, N., and R. Zhong. 1999. “An Approximate Formula for Pricing American Options.” The Journal of Derivatives 7 (2): 31-40. doi:10.3905/jod.1999.319140.

Kim, I. 1990. “The Analytic Valuation of American Options." Review of Financial Studies 3 (4): 547-572. doi:10.1093/rfs/3.4.547.

Kirk, E. 1996. “Correlation in Energy Markets.” In Managing Energy Price Risk, 71-78. London: RISK Books.

Li, M., S. J. Deng, and J. Zhou. 2008. "Closed-Form Approximations for Spread Option Prices and Greeks.” The Journal of Derivatives 15 (3): 58-80. doi:10.3905/jod.2008.702506.

Li, M., S. J. Deng, and J. Zhou. 2010. "Multi-Asset Spread Option Pricing and Hedging." Quantitative Finance 10 (3): 305-324. doi:10.1080/14697680802626323.

Lim, G. C., G. M. Martin, and V. L. Martin. 2005. "Parametric Pricing of Higher Order Moments in S\&P500 Options." Journal of Applied Econometrics 20 (3): 377-404. doi:10.1002/(ISSN) 1099-1255.

Longstaff, F. A., and E. S. Schwartz. 2001. "Valuing American Options by Simulation: A Simple Least-Squares Approach.” The Review of Financial Studies 14: 113-147. doi:10.1093/rfs/ 14.1.113.

Margrabe, W. 1978. "The Value of an Option to Exchange One Asset for Another." The Journal of Finance 33 (1): 177-186.

Merton, R. C. 1976. "Option Pricing When Underlying Stock Returns are Discontinuous." Journal of Financial Economics 3: 125-144.

Neuberger, A. 2012. "Realized Skewness." Review of Financial Studies 25 (11): 3423-3455. doi:10.1093/rfs/hhs101.

Poitras, G. 1998. Spread Options, Exchange Options, and Arithmetic Brownian Motion. Journal of Futures Markets 18 (5): 487-517. cited By (since 1996)12 doi:10.1002/(ISSN)1096-9934.

Rompolis, L. S. 2010. "Retrieving Risk Neutral Densities from European Option Prices Based on the Principle of Maximum Entropy." Journal of Empirical Finance 17 (5): 918-937. doi:10.1016/j.jempfin.2010.04.007.

Rompolis, L. S., and E. Tzavalis. 2008. "Recovering Risk Neutral Densities from Option Prices: A New Approach.” Journal of Financial and Quantitative Analysis 43 (4): 1037-1054. doi:10.1017/S0022109000014435.

Rubinstein, M. 1994. “Implied Binomial Trees.” The Journal of Finance 49 (3): 771-818. doi: 10.1111/j.1540-6261.1994.tb00079.x

Rubinstein, M. 1998. "Edgeworth Binomial Trees." The Journal of Derivatives 5 (3): 20-27. doi:10.3905/jod.1998.407994.

Schlögl, E. 2013. "Option Pricing Where the Underlying Assets Follow a Gram/Charlier Density of Arbitrary Order.” Journal of Economic Dynamics \& Control 37: 611-632. doi:10.1016/j. jedc.2012.10.001.

Schoutens, W. 2005. “Moment Swaps." Quantitative Finance 5 (6): 525-530. doi:10.1080/ 14697680500401490.

Shimko, D. 1994. “Options on Futures Spreads: Hedging, Speculation, and Valuation.” Journal of Futures Markets 14 (2): 183-213. doi:10.1002/(ISSN)1096-9934.

Tallis, G. M. 1965. "Plane Truncation in Normal Populations." Journal of the Royal Statistical Society. Series B (Method- Ological) 27 (2): 301-307.

Tian, Y. 2011. "Extracting Risk-Neutral Density and Its Moments from American Option Prices." The Journal of Derivatives 18 (3): 17-34. doi:10.3905/jod.2011.18.3.017.

Zhang, J. E., and Y. Xiang. 2008. “The Implied Volatility Smirk.” Quantitative Finance 8 (3): 263284. doi:10.1080/14697680601173444.

Zhao, H., J. E. Zhang, and E. C. Chang. 2013. “"The Relation between Physical and Risk-Neutral Cumulants.” International Review of Finance 13 (3): 345-381. doi:10.1111/irfi.2013.13.issue-3. 


\section{Appendix A. Analytical proofs}

\section{A.1 Proof of Proposition 2.1}

Equation (11) was calculated as an approximation in Section 4 of Arismendi (2014). To get the equality, we need to prove that

$$
\sum_{l_{1}=1}^{n} \sum_{l_{2}=1}^{n} \frac{1}{2}\left(1-\lambda_{\operatorname{VCF}(2)}\right) k_{l_{1}, l_{2}}\left(g_{\mathbf{S}(t)}\right) \frac{\partial^{2}}{\partial s_{l_{1}} \partial s_{l_{2}}} g_{\mathbf{S}}=\sum_{j=3}^{\infty} M_{\left[l_{1},\left[l_{2},\left[\ldots,\left[l_{j}\right] \ldots\right]\right.\right.} \frac{(-1)^{j}}{j !} \frac{\partial^{j}}{\partial s_{\left[l_{1}\right]} \ldots \partial s_{\left[l_{j}\right]}} g_{\mathbf{S}}=\varepsilon(\mathbf{s}, 3),
$$

but as the series is convergent, then

$$
\sum_{l_{1}=1}^{n} \sum_{l_{2}=1}^{n} \frac{1}{2}\left(1-\lambda_{\mathrm{VCF}(2)}\right) k_{l_{1}, l_{2}}\left(g_{\mathbf{S}(t)}\right) \frac{\partial^{2}}{\partial s_{l_{1}} \partial s_{l_{2}}} g_{\mathbf{S}}=\varepsilon(\mathbf{s}, 3)<\infty .
$$

\section{A.2 Proof of Proposition 4.2}

Applying the definition of the MVLN, and after the change of variable $S_{i}=\exp \left(Y_{i}\right)$, we have the resulting moment over the MVN distribution:

$$
\begin{aligned}
& \mathbb{E}_{0}^{Q}\left[\log \left(S_{1}(t)\right)^{\alpha_{1}} \ldots \log \left(S_{n}(t)\right)^{\alpha_{n}} S_{1}(t)^{\beta_{1}} \ldots S_{n}(t)^{\beta_{n}} \mid S_{j}(t) \geq K\right] \\
& =L_{1}^{-1}\left({ }^{(n-1)} \int_{0}^{\infty} \int_{K}^{\infty}(2 \pi)^{-n / 2}\left|\Sigma_{\mathbf{s}}\right|^{-1 / 2}\left(\prod_{i=1}^{n} \log \left(s_{i}\right)^{\alpha_{i}}\right)\left(\prod_{i=1}^{n} s_{i}^{\beta_{i}-1}\right)\right. \\
& \left.\times \exp \left(-\frac{1}{2}\left(\log (\mathbf{s})-\mu_{\mathbf{s}}\right)^{\prime} \Sigma_{\mathbf{s}}^{-1}\left(\log (\mathbf{s})-\mu_{\mathbf{s}}\right)\right) d \mathbf{s}\right) \\
& =L_{1}^{-1}\left({ }^{(n-1)} \int_{-\infty}^{\infty} \int_{\log (K)}^{\infty}(2 \pi)^{-n / 2}\left|\Sigma_{\mathbf{s}}\right|^{-1 / 2}\left(\prod_{i=1}^{n} y_{i}^{\alpha_{i}}\right)\right. \\
& \left.\times \exp \left(\mathbf{y}^{\prime} \beta\right) \exp \left(-\frac{1}{2}\left(\mathbf{y}-\mu_{\mathbf{s}}\right)^{\prime} \Sigma_{\mathbf{s}}^{-1}\left(\mathbf{y}-\mu_{\mathbf{s}}\right)\right) d \mathbf{y}\right)
\end{aligned}
$$

where $L_{1}=\mathbb{P}^{Q}\left(S_{j}(t) \geq K\right)$. The time parameter of the process $\mathbf{Y}(t)$ is omitted to simplify the notation. The last expression can be transformed as

$$
\begin{aligned}
& =L_{1}^{-1}\left({ }^{(n-1)} \int_{-\infty}^{\infty} \int_{\log (K)}^{\infty}(2 \pi)^{-n / 2}\left|\Sigma_{\mathbf{s}}\right|^{-1 / 2}\left(\prod_{i=1}^{n} y_{i}^{\alpha_{i}}\right)\right. \\
& \left.\times \exp \left(\frac{1}{2} \beta^{\prime} \Sigma_{\mathbf{s}} \beta+\beta^{\prime} \mu_{\mathbf{s}}-\frac{1}{2}(\mathbf{y}-\zeta)^{\prime} \Sigma_{\mathbf{s}}^{-1}(\mathbf{y}-\zeta)\right) \mathrm{d} \mathbf{y}\right)
\end{aligned}
$$

with $\zeta=\mu_{\mathrm{s}}+\Sigma_{\mathrm{s}} \beta$. Define $L_{2}=\mathbb{P}^{Q}\left(Y_{j}(t) \geq \log (K)\right)$, then the last expression becomes

$$
=\exp \left(\frac{1}{2} \beta^{\prime} \Sigma_{\mathbf{s}} \beta+\beta^{\prime} \mu_{\mathrm{s}}\right)\left(\frac{L_{2}}{L_{1}}\right) \mathbb{E}_{0}^{Q}\left[Y_{1}(t)^{\alpha_{1}} \ldots Y_{n}(t)^{\alpha_{n}} \mid Y_{j}(t) \geq \log (K)\right]
$$

Then, the variable $\mathbf{Y}(t)$ is distributed $N\left(\mu_{\mathbf{s}}+\Sigma_{\mathbf{s}} \beta, \Sigma_{\mathbf{s}}\right)$, the constant $A=$ $\exp \left(\frac{1}{2} \beta^{\prime} \Sigma_{\mathbf{s}} \beta+\beta^{\prime} \mu_{\mathbf{s}}\right)\left(\frac{L_{2}}{L_{1}}\right)$ and the result follows. 


\section{A.3 Proof of Proposition 4.3}

Setting the change of variable $S_{i}=\exp \left(Y_{i}\right)$, and following the same steps as in the demonstration of Proposition 4.2, we reach the result:

$$
\begin{aligned}
\mathbb{E}_{0}^{Q} & {\left[\log \left(S_{1}(t)\right)^{\alpha_{1}} \ldots \log \left(S_{n}(t)\right)^{\alpha_{n}} S_{1}(t)^{\beta_{1}} \ldots S_{n}(t)^{\beta_{n}} \mid S_{j}(t) \geq S_{i}(t)\right] } \\
& =\exp \left(\frac{1}{2} \beta^{\prime} \Sigma_{\mathbf{s}} \beta+\beta^{\prime} \mu_{\mathrm{s}}\right)\left(\frac{L_{2}}{L_{1}}\right) \mathbb{E}_{0}^{Q}\left[Y_{1}(t)^{\alpha_{1}} \ldots Y_{n}(t)^{\alpha_{n}} \mid Y_{j}(t) \geq Y_{i}(t)\right],
\end{aligned}
$$

where $L_{1}=\mathbb{P}^{Q}\left(S_{j}(t) \geq S_{i}(t)\right), L_{2}=\mathbb{P}^{Q}\left(Y_{j}(t) \geq Y_{i}(t)\right)$, the variable $\mathbf{Y}(t)$ is distributed $N\left(\mu_{\mathbf{s}}+\Sigma_{\mathrm{s}} \beta, \Sigma_{\mathrm{s}}\right)$, the constant $A=\exp \left((1 / 2) \beta^{\prime} \Sigma_{\mathbf{s}} \beta+\beta^{\prime} \mu_{\mathbf{s}}\right)\left(L_{2} / L_{1}\right)$. The first two moments of the variable $\mathbf{Y}(t)$ truncated at plane $B_{\mathbf{Y}(t)}$ are derived from the results of Tallis (1965). For this case, the variable $\mathbf{Y}(t)$ is non-standard normal, therefore, the variable must be standardized in the plane restriction. The density function of the variable $\mathbf{Y}(t)$ is

$$
f_{\mathbf{y}(t)}=(2 \pi)^{-n / 2}\left|\Sigma_{\mathbf{s}}\right|^{-1 / 2} \exp \left(-\frac{1}{2}\left(\mathbf{y}(t)-\mu_{\mathbf{s}}\right) 1 S_{\mathbf{s}}^{-1}\left(\mathbf{y}(t)-\mu_{\mathbf{s}}\right)\right) .
$$

Let $\mathbf{D}=\operatorname{diag}\left(\sigma_{1}, \ldots, \sigma_{n}\right)$ be a diagonal matrix with the volatility of $\Sigma_{\mathbf{s}}$ in the diagonal. The $p d f$ of (33) could be expressed as

$$
\begin{aligned}
f_{\mathbf{y}(t)} & =(2 \pi)^{-n / 2}\left|\Sigma_{\mathbf{s}}\right|^{-1 / 2} \exp \left(-\frac{1}{2}\left(\mathbf{y}(t)-\mu_{\mathbf{s}}\right)\left(\mathbf{D}^{\prime} \mathbf{R D}\right)^{-1}\left(\mathbf{y}(t)-\mu_{\mathbf{s}}\right)\right) \\
& =(2 \pi)^{-n / 2}\left|\Sigma_{\mathbf{s}}\right|^{-1 / 2} \exp \left(-\frac{1}{2} \mathbf{z}(t)^{\prime}(\mathbf{R})^{-1} \mathbf{z}(t)\right),
\end{aligned}
$$

where $\mathbf{Y}(t)=\mathbf{Z}(t)^{\prime} \mathbf{D}+\mu_{\mathbf{s}}$, hence:

$$
\begin{aligned}
\mathbf{b}^{\prime} \mathbf{Y}(t) \geq p_{1} & \Rightarrow \mathbf{b}^{\prime}\left(\mathbf{Z}(t)^{\prime} \mathbf{D}+\mu_{\mathrm{s}}\right) \geq p_{1} \\
& \Rightarrow \mathbf{b}^{\prime}\left(\mathbf{Z}(t)^{\prime} \mathbf{D}\right)+\mathbf{b}^{\prime} \mu_{\mathbf{s}} \geq p_{1} \\
& \Rightarrow\left(\mathbf{b}^{\prime} \mathbf{D}\right) \mathbf{Z}(t) \geq p_{1}-\mathbf{b}^{\prime} \mu_{\mathbf{s}}
\end{aligned}
$$

and the result follows from the formula of Tallis1965 adding $\mu_{\mathbf{s}}+\Sigma_{\mathbf{s}} \beta$ to the first moment.

\section{Appendix B. Bivariate option pricing with closed-form truncated moments}

\section{B.1 Exchange option price using multivariate truncated moments}

Define two assets with GBM price processes $S_{1}(t), S_{2}(t)$ as in (8), with $\sigma_{1}, \sigma_{2}$ their corresponding constant volatilities, denote by $S(t)=S_{1}(t) / S_{2}(t)$. The exchange option is a contract with payoff:

$$
\Pi\left(S_{1}(t), S_{2}(t), K\right)=\left[S_{1}(t)-S_{2}(t)\right]^{+} .
$$

Applying a change of numeraire technique, the price of the option is

$$
C_{0}\left(\Pi\left(S_{1}(t), S_{2}(t), K\right)\right)=\exp (-r t) S_{2}(0) \mathbb{P}^{Q}(S(t) \geq 1)\left(\mathbb{E}_{0}^{Q}[S(t) \mid S(t) \geq 1]-1\right) .
$$


Applying Proposition 4.1, we find the value of $\mathbb{E}_{0}^{Q}[S(t) \mid S(t) \geq 1]$, and the value of $P^{Q}(S(t) \geq 1)$ where

$$
S(t) \sim L N(\mu, V),
$$

with $\mu=\log \left(S_{1}(0) / S_{2}(0)\right)-(1 / 2) V, V=\left(\sigma_{1}^{2}+\sigma_{2}^{2}-2 \rho \sigma_{1} \sigma_{2}\right) t$. Because the solution has been provided using a change of numeraire, this is still a univariate density solution.

\section{B.2 Spread option price using multivariate truncated moments}

Consider two assets $S_{1}(t), S_{2}(t)$ defined as in Section B.1. The payoff contract of a spread option is defined as

$$
\Pi\left(S_{1}(t), S_{2}(t), K\right)=\left[S_{1}(t)-S_{2}(t)-K\right]^{+} .
$$

The option price could be calculated as

$$
C_{0}\left(\Pi\left(S_{1}(t), S_{2}(t), K\right)\right)=\exp (-r t) \mathbb{E}_{0}^{Q}\left[\max \left(S_{1}(T)-S_{2}(T)-K, 0\right)\right] .
$$

A closed-form formula for this option is unknown. The Kirk1996 approximation uses the Margrabe1978 result on exchange options (change of numeraire) jointly with an approximation of the distribution of the asset $S_{2}(t)$ plus the strike value $K$ :

$$
\left(S_{2}(t)+K\right)=S_{a}(t) \sim L N\left(\log \left(S_{2}(0)+K \exp (-r t)\right)+\left(\left(r-\frac{1}{2} \sigma_{2}^{2}\right) t,\left(\sigma_{2} F_{2}\right)^{2} t\right),\right.
$$

where $F_{2}=S_{2}(0) / S_{2}(0)+K \exp (-r t)$ is the volatility approximation. Using this distribution approximation, the payoff transforms into

$$
\Pi\left(S_{1}(t), S_{2}(t), K\right)=\left[S_{1}(t)-S_{a}(t)\right]^{+},
$$

which could be considered as an exchange option and could be solved as in Section B.1: Define $S(t)=S_{1}(t) / S_{a}(t)$, and with the notation of truncated moments, the value of the option is

$$
C_{0}\left(\Pi\left(S_{1}(t), S_{2}(t), K\right)\right)=\exp (-r t)\left(S_{2}(0)+K \exp (-r t)\right) \mathbb{P}^{Q}(S(t) \geq 1)\left(\mathbb{E}_{0}^{Q}[S(t) \mid S(t) \geq 1]-1\right) .
$$

Applying Proposition 4.1, we find the value of $\mathbb{E}_{0}^{Q}[S(t) \mid S(t) \geq 1]$, and the value of $\mathbb{P}^{Q}(S(t) \geq 1)$ where

$$
S(t) \sim L N(\mu, V)
$$

with $\mu=\log \left(S_{1}(0) /\left(S_{2}(0)+K \exp (-r t)\right)\right)-(1 / 2) V, V=\left(\sigma_{1}^{2}+\left(\sigma_{2} F_{2}\right)^{2}-2 \rho \sigma_{1} \sigma_{2} F_{2}\right) t$. 


\section{Appendix C}

Table C1. MGEE basket option price approximation of American put options using an implied American risk-neutral density.

\begin{tabular}{|c|c|c|c|c|c|c|c|c|c|c|}
\hline \multirow[b]{2}{*}{$t$} & \multicolumn{4}{|c|}{ Multivariate GBM processes } & \multicolumn{3}{|c|}{ Li2010 } & \multicolumn{3}{|c|}{ AV2012 } \\
\hline & $K$ & $r$ & American & European & VCF(W) & $\operatorname{VCF}(1)$ & $\operatorname{VCF}(2)$ & VCF(W) & $\operatorname{VCF}(2)$ & $\operatorname{VCF}(2)$ \\
\hline \multirow{5}{*}{0.25} & 95 & 0.05 & 0.239 & 0.230 & 0.498 & 0.258 & 0.513 & 0.824 & 0.583 & 0.848 \\
\hline & & 0.10 & 0.148 & 0.131 & 0.498 & 0.290 & 0.575 & 0.824 & 0.643 & 0.995 \\
\hline & 100 & 0.05 & 1.532 & 1.424 & 0.500 & 0.074 & - & 0.955 & 0.640 & 0.603 \\
\hline & & 0.10 & 1.210 & 0.976 & 0.499 & 0.132 & 0.262 & 0.954 & 0.801 & 0.682 \\
\hline & 105 & 0.05 & 4.945 & 4.396 & 0.501 & 0.264 & 1.065 & 0.737 & 1.023 & 1.535 \\
\hline \multirow{7}{*}{1.00} & & 0.10 & 4.745 & 3.452 & 0.501 & 0.184 & 1.338 & 0.738 & 1.245 & 1.890 \\
\hline & 95 & 0.05 & 0.911 & 0.772 & 0.500 & 0.481 & 0.496 & 0.931 & 0.687 & 0.984 \\
\hline & & 0.10 & 0.434 & 0.260 & 0.499 & 0.485 & 0.497 & 0.915 & 0.843 & 0.995 \\
\hline & 100 & 0.05 & 2.424 & 1.939 & 0.501 & 0.485 & 0.485 & 0.955 & 0.843 & 0.671 \\
\hline & & 0.10 & 1.594 & 0.793 & 0.500 & 0.484 & 0.449 & 0.944 & 1.107 & 0.519 \\
\hline & 105 & 0.05 & 5.311 & 3.950 & 0.503 & 0.492 & 0.298 & 0.886 & 1.109 & 1.442 \\
\hline & & 0.10 & 4.733 & 1.901 & 0.501 & 0.485 & 0.247 & 0.881 & 1.428 & 2.045 \\
\hline
\end{tabular}

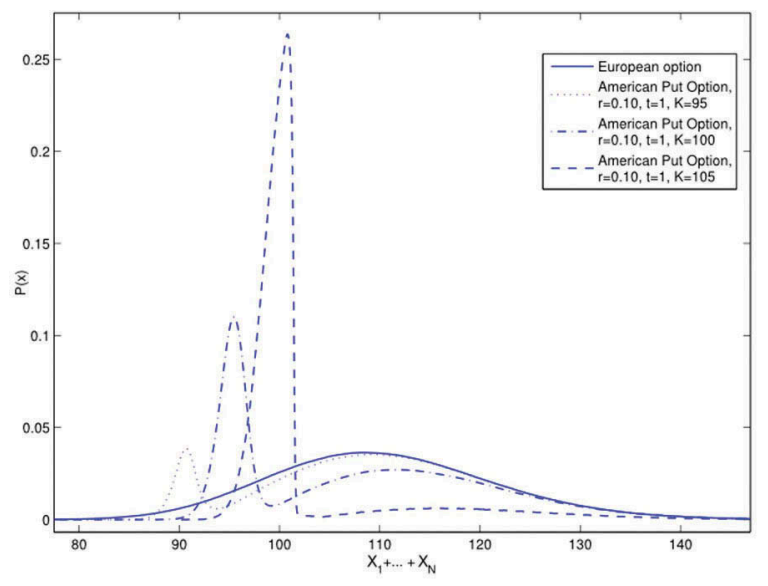

Figure C1. Empirical American option implied density of $X_{1}+\cdots+X_{N}$. (a) Axis S1 and S2 of the European risk-neutral density $g S(t)$ (darker) and the American option implied density (lighter) $f X(t)$, adjusted only by the first-order moment differences. (b) Axis S1 and S2 of the European risk-neutral density $g S(t)$ (darker) and the American option implied density (lighter) $f \chi(t)$, adjusted by the firstand second-order moment differences. (c) Difference of the risk-neutral density in C2(a), effects of the early exercise premium. 


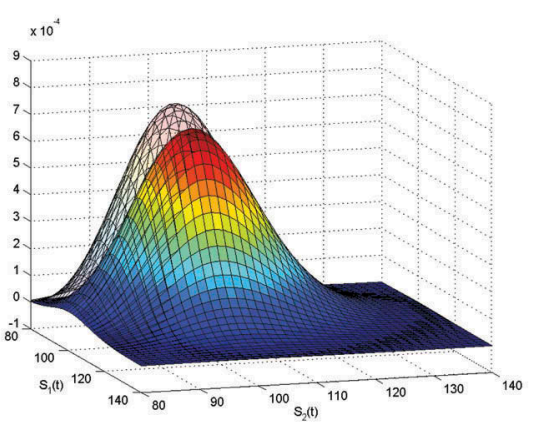

(a)

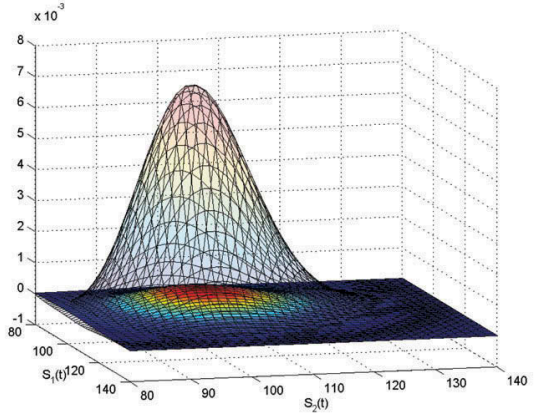

(b)

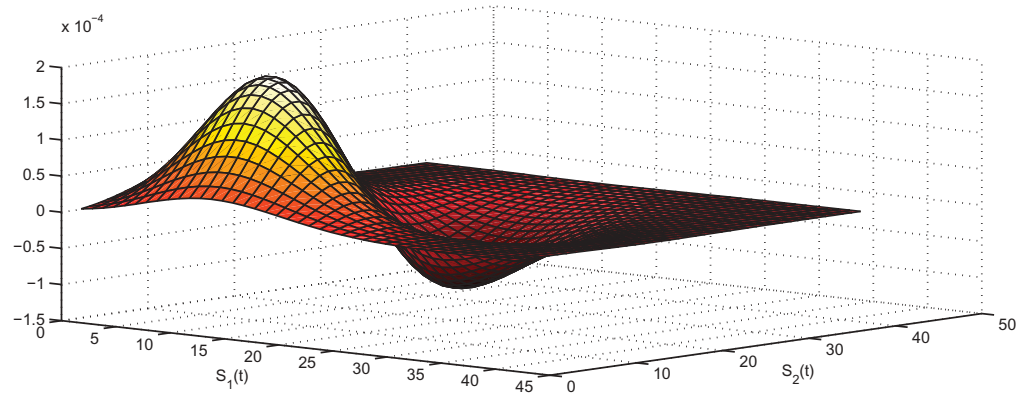

(c)

Figure C2. European and American implied risk-neutral densities for GBM diffusions. (a) Axis S1 and $S 2$ of the European risk-neutral density $g S(t)$ (darker) and the American option implied density (lighter)fX(t), adjusted only by the first-order moment differences from a jump-diffusion process. (b) Axis $S 1$ and $S 2$ of the European risk-neutral density $g S(t)$ (darker) and the American option implied density (lighter) $f x(t)$, adjusted by the first- and second-order moment differences from a jumpdiffusion process.

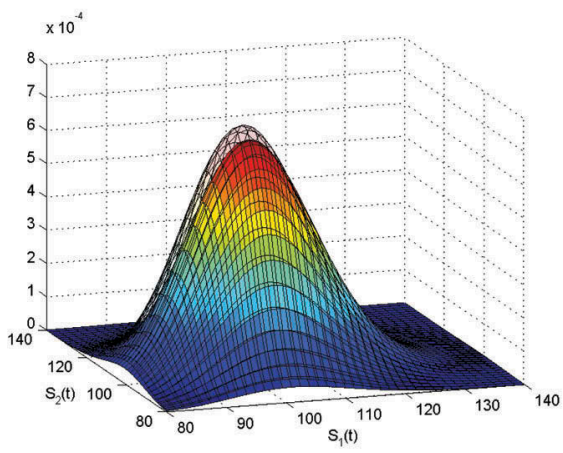

(a)

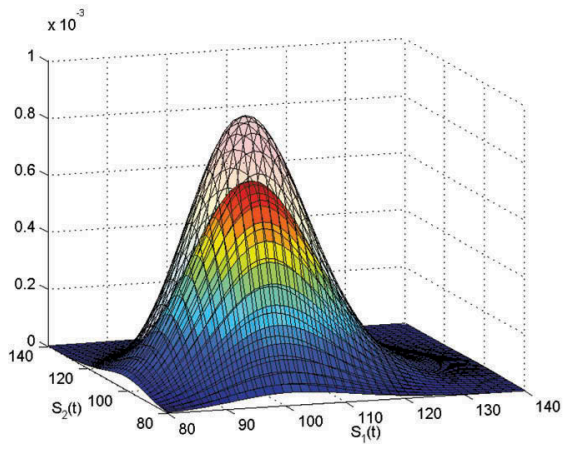

(b)

Figure C3. European and American implied risk-neutral densities for multivariate jump-diffusion processes. 